\title{
BEGINNING OF STABILITY THEORY FOR POLISH SPACES SH849
}

\author{
SAHARON SHELAH
}

\begin{abstract}
We consider stability theory for Polish spaces and more generally for definable structures (say with elements of a set of reals). We clarify by proving some equivalent conditions for $\aleph_{0}$-stability. We succeed to prove existence of indiscernibles under reasonable conditions; this gives strong evidence that such a theory exists.
\end{abstract}

Version 2017-04-07_12. See https://shelah.logic.at/papers/849/ for possible updates.

Date: April 7, 2017.

2010 Mathematics Subject Classification. Primary: 03C45, 03E15; Secondary: 03C57, 03C75.

Key words and phrases. model theory, descriptive set theory, classification theory, number of non-isomorphic models, indiscernible sets, categoricity, Polish structures.

The author would like to thank the Israel Science Foundation for partial support of this research (Grant No. 242/03). This was part of [She11] (first version 29/March/2001) which was submitted 17/7/2002; but separated in Feb. 2009. First version - 09/Feb/13. 


\section{$\S 0$. INTRODUCTION}

\section{$\S 0(\mathrm{~A})$. General Aims.}

Question 0.1. Is there a stability theory/classification theory of Polish spaces/algebras (more generally definable structures say on the continuum)?

Naturally we would like to develop a parallel to classification theory and in particular stability theory (see [She90]). A natural test problem is to generalize "Morley theorem = Eos conjecture". But we only have one model so does it mean anything?

On way is to restrict ourselves to a class of e.g. abelian groups and replace "categorical" by "free"; this is reasonable as a free algebra is unique in a strong sense (determined up to isomorphism by its dimension which is equal to its cardinality when uncountable, well when the vocabulary is countable).

We may consider any variety or just a theory consisting of universal Horn sentences (as then being a free algebra is well defined); this is an interesting question per se. But still this is a special case; and in general, we may change the universe.

Example 0.2. If $\mathbb{P}$ is adding $\left(2^{\aleph_{0}}\right)^{+}$-Cohen subsets of $\omega$ then

$$
(\mathbb{C})^{\mathbf{V}} \text { and }(\mathbb{C})^{\mathbf{V}[\mathbb{P}]}
$$

are both algebraically closed fields of characteristic 0 which are not isomorphic (as they have different cardinalities).

So we restrict ourselves to forcing notions $\mathbb{P}_{1} \lessdot \mathbb{P}_{2}$ such that

$$
\left(2^{\aleph_{0}}\right)^{\mathbf{V}\left[\mathbb{P}_{1}\right]}=\left(2^{\aleph_{0}}\right)^{\mathbf{V}\left[\mathbb{P}_{2}\right]}
$$

and compare the Polish models in $\mathbf{V}^{\mathbb{P}_{1}}, \mathbf{V}^{\mathbb{P}_{2}}$. We may restrict our forcing notions to c.c.c. or whatever.

Example 0.3. Under any such interpretation

(a) $\mathbb{C}=$ the field of complex numbers is categorical

(b) $\mathbb{R}=$ the field of the reals is not (by adding $2^{\aleph_{0}}$ many Cohen reals).

(Why? Say we assume $\mathbb{P}_{1} \lessdot \mathbb{P}_{2},\left(2^{\aleph_{0}}\right)^{\mathbf{V}\left[\mathbb{P}_{1}\right]}=\left(2^{\aleph_{0}}\right)^{\mathbf{V}\left[\mathbb{P}_{2}\right]}$ but $\mathbb{R}^{\mathbf{V}\left[\mathbb{P}_{1}\right]} \neq \mathbb{R}^{\mathbf{V}\left[\mathbb{P}_{2}\right]}$. Trivially: $\mathbb{R}^{\mathbf{V}\left[\mathbb{P}_{2}\right]}$ is complete in $\mathbf{V}\left[\mathbb{P}_{2}\right]$ while $\mathbb{R}^{\mathbf{V}\left[\mathbb{P}_{1}\right]}$ in $\mathbf{V}^{\left[\mathbb{P}_{2}\right]}$ is not complete, but there are less trivial reasons).

Conjecture 0.4. We have a dichotomy for "reasonable definable model" (e.g. Polish or Suslin ones), i.e. either the model is similar to categorical theories, or there are "many complicated models" under the present interpretation.

So in particular we expect the natural variants of central notions defined below (like categoricity) will be equivalent; in particular we expect that it will be enough to consider the forcing notions of adding Cohen reals. Naturally those questions call for the use of descriptive set theory on the one hand and model theory on the other hand; in particular to using definability in both senses and using $\mathbb{L}_{\aleph_{1}, \aleph_{0}}(\mathbf{Q})$.

Presently, i.e. here there is no serious use of either; the questions are naturally inspired by model theory. It would be natural to consider questions inspired by the investigation of such specific structures; to some extent considering the freeness of a definable Abelian group falls under this. 
A priori, trying to connect different directions in mathematics is tempting, but it may well lack non-trivial results. We suggest that the result on the existence of indiscernibility, 3.8, give serious evidence that this is not the case here; note that even the weaker 1.7 gives more than set theory, i.e. Erdös-Rado theorem. That is, the question is: is there a non-trivial theory in this direction? While the present work does not achieve a real theory of this kind, we believe that it gives an existence proof.

Let us elaborate suggestions for the definition of "categorical" and for "definable". Recall that it is well known that: a Polish structure is an $F_{\sigma}$-one which is a Borel one; a Borel (set or structure) is a $\Sigma_{1}^{1}$-one, a $\Sigma_{1}^{1}$-set/structure is a $\Sigma_{2}^{1}$-one and a $\Sigma_{2}^{1}$-set/structure is a $\aleph_{1}$-Suslin one, see e.g. [Jec03].

Definition 0.5. 1) Let $\mathfrak{A}$ denote a definition of a $\tau$-structure, $\tau$ a countable vocabulary, the set of elements of the structure is the reals or a definable set of reals such that it is absolute enough, i.e. for any forcing notion $\mathbb{P} \lessdot \mathbb{Q}$ we have $\mathfrak{A}\left[\mathbf{V}^{\mathbb{P}}\right] \subseteq \mathfrak{A}\left[\mathbf{V}^{\mathbb{Q}}\right]$ but we may say "the structure/model $\mathfrak{A}$ ".

2) We say $\mathfrak{A}$ is $F_{\sigma} /$ Borel $/ \Sigma_{1}^{1} / \kappa$-Suslin, etc., if the definition mentioned above is $F_{\sigma} /$ Borel $/ \Sigma_{1}^{1} / \kappa$-Suslin, etc.

3) We say $\mathfrak{A}$ is categorical 1 when for any forcing notions $\mathbb{P} \lessdot \mathbb{Q}$ such that $\Vdash$ " $\left(2^{\aleph_{0}}\right)^{\mathbf{V}[\mathbb{P}]}=\left(2^{\aleph_{0}}\right)^{\mathbf{V}[\mathbb{Q}] " ~ t h e ~ s t r u c t u r e s ~} \mathfrak{A}\left[\mathbf{V}^{\mathbb{P}}\right], \mathfrak{A}\left[\mathbf{V}^{\mathbb{Q}}\right]$ are isomorphic in $\mathbf{V}^{\mathbb{Q}}$.

$3 \mathrm{~A}$ ) We say $\mathfrak{A}$ is categorical 1 under $\varphi$ (e.g. $\varphi=\left(2^{\aleph_{0}}=\aleph_{1}\right)$ means that for any forcing notions $\mathbb{P} \lessdot \mathbb{Q}$ satisfying $\Vdash_{\mathbb{P}} " \varphi$ ", $\Vdash_{\mathbb{Q}}$ " $\varphi$ " and $\Vdash_{\mathbb{Q}} "\left(2^{\aleph_{0}}\right) \mathbf{V}[\mathbb{P}]=\left(2^{\aleph_{0}}\right) \mathbf{V}[\mathbb{Q}]$ " the structures $\mathfrak{A}\left[\mathbf{V}^{\mathbb{P}}\right], \mathfrak{A}\left[\mathbf{V}^{\mathbb{Q}}\right]$ are isomorphic in $\mathbf{V}^{\mathbb{Q}}$.

4) We say $\mathfrak{A}$ is $\mathfrak{K}$-categorical ${ }_{1}$ when above $\mathbb{P}, \mathbb{Q} \in \mathfrak{K}$ (or the pair $(\mathbb{P}, \mathbb{Q}) \in \mathfrak{K}$ ).

5) If $\lambda$ is a cardinal or a definition of a cardinal then $\mathfrak{A}$ is categorical ${ }_{1}$ in $\lambda$, is defined as in $(3 \mathrm{~A})$ but $\Vdash_{\mathbb{P}}$ " $\varphi$ ", $\Vdash_{\mathbb{Q}}$ " $\varphi$ " is replaced by $\lambda\left[\mathbf{V}^{\mathbb{P}}\right]=\lambda\left[\mathbf{V}^{\mathbb{Q}}\right]=\left(2^{\aleph_{0}}\right) \mathbf{V}[\mathbb{P}]=$ $\left(2^{\aleph_{0}}\right)^{\mathbf{V}[\mathbb{Q}]}$ and this is a non-empty condition; similarly in $(3 \mathrm{~A}),(4)$.

6) Let $T$ be a set of (first order) equations in the countable vocabulary $\tau$. Let $\mathfrak{A}$ be a model of $T$. We say $\mathfrak{A}$ is free ${ }_{1}$ for $\mathfrak{K}$ when for every $\mathbb{P} \in \mathfrak{K}$ the model $\mathfrak{A}\left[\mathbf{V}^{\mathbb{P}}\right]$ is a free algebra. Similarly, parallely to $(3 \mathrm{~A}),(4),(5)$.

Conjecture 0.6. 1) If, e.g. a $\Sigma_{1}^{1}$-structure $\mathfrak{A}$ is categorical 1 in some $\aleph_{\alpha} \geq \aleph_{\omega}$ for $\mathfrak{K}$ then it is categorical in every $\aleph_{\alpha} \geq \aleph_{\omega}$ of cofinality $>\aleph_{0}$ for $\mathfrak{K}$ where $\mathfrak{K}=\left\{(\mathbb{P}, \mathbb{Q}): \mathbb{P} \lessdot \mathbb{Q}\right.$ and $\left(2^{\aleph_{0}}\right)\left[\mathbf{V}^{\mathbb{P}}\right]=\left(2^{\aleph_{0}}\right)\left[\mathbf{V}^{\mathbb{Q}}\right] \geq \aleph_{\omega}\left[\mathbf{V}^{\mathbb{P}}\right]$, Card $\left.\left[\mathbf{V}^{\mathbb{P}}\right]=\operatorname{Card}\left[\mathbf{V}^{\mathbb{Q}}\right]\right\}$. 2) Or at least "for every $\aleph_{\alpha} \geq \aleph_{\omega_{1}}$ " and/or restricting the forcing notion to be c.c.c. 3) Similarly for freeness.

Thesis 0.7. 1) Classification theory for such models resembles more the case of $\mathbb{L}_{\omega_{1}, \omega}$ than the first order.

2 ) If the continuum is too small we may get categoricity for "incidental" reasons.

3) In our context the parallel of categoricity and "being in the low side of the main gap" seems to be equal.

See [She09b]; as support for this thesis, in [She11, §5] we prove:

Theorem 0.8. 1) There is an $F_{\sigma}$-abelian group $G$ (i.e. an $F_{\sigma}$-definition, in fact a very explicit definition) such that $\mathbf{V} \models$ " $G$ is a free abelian group" iff $\mathbf{V} \models 2^{\aleph_{0}}<$ $\aleph_{736}$.

2) Moreover, it follows that if $\mathbf{V} \models$ "G is not free" and $\mathbb{P}$ is a c.c.c. forcing notion then the abelian group $G^{\mathbf{V}}$ is not free even in $\mathbf{V}^{\mathbb{P}}$. 
Comments: In the context of the previous theorem we cannot do better than $F_{\sigma}$, but we may hope for some other examples which is not a group or categoricity is not because of freeness.

The proof gives

Conclusion 0.9. For any $n<\omega$ for some $F_{\sigma}$-abelian group $\mathfrak{A}, \mathfrak{A}$ is categorical cin $_{1}$ $\aleph_{\alpha}$ iff $\alpha \leq n$.

Note that if $\operatorname{cf}(\alpha)=\aleph_{0}$ so necessarily $\alpha>n$, then there are no forcing notions $\mathbb{P}, \mathbb{Q}$ as in $0.5(5)$, hence categoricity fails by the definition.

A connection with the model theory is that by Hart-Shelah [HS90] such things can also occur in $\mathbb{L}_{\omega_{1}, \omega}$ whereas (by [She83a], [She83b]) if $\bigwedge\left(2^{\aleph_{n}}<2^{\aleph_{n+1}}\right.$ ) and $\psi \in \mathbb{L}_{\omega_{1}, \omega}$ is categorical in every $\aleph_{n}$, then $\psi$ is categorical in every $\lambda$. See more in Shelah-Villaveces [SV21] and in [She09a]. We may consider other interpretations of "categoricity", more on $0.7-0.9$, see $\S(0 \mathrm{C})$ and $\S 4$.

The parallels here are still open.

Those questions may cast some light on the thesis that non-first order logics are "more distant" from the "so-called" mainstream mathematics.

This work originally was a section in [She11]; in it we try to look at stability theory in this context, proving the modest (in 3.8):

$\boxplus$ for " $\aleph_{0}$-stable $\kappa$-Suslin models" the theorem on the existence of indiscernibles can be generalized.

A natural question is whether in the existence of indiscernibles (in Theorem 3.8) we can start with a set of cardinality $\lambda$ rather than $\lambda^{+\kappa^{+}}$. Another natural question is whether categoricity implies that the $\mathbb{L}_{\infty, \aleph_{0}}$-theory of $\mathfrak{A}$ is "simple", clearly by $\S(0 \mathrm{~A})$ we are sure this holds.

Both questions (and maybe the one on the right parallel of stable) may be addressed in a work in progress, $\left[\mathrm{S}^{+}\right]$.

We thank Udi Hrushovski and the referee for many helpful comments.

\section{$\S 0(\mathrm{~B})$. The Content of the Present Work.}

Our context is a $\kappa$-candidate $(\mathfrak{A}, \Delta)$, so $\mathfrak{A}$ is structure with the set of elements being a sets of reals, which is reasonably definable, and to some extent similarly the relations the functions of $\mathfrak{A}$ from $\Delta$ being reasonably definable: usually $\kappa$-Suslin; you can fix $\Delta$ as the set of quantifier free formulas. In the present work forcing does not appear, so the universe is fixed.

In $\S 1$; we give some basic definitions in Definition 1.4 but note that the $\aleph_{0}$-stable and $\aleph_{0}$-unstable are proved to be complimentary only later. The main result is to get the "end-extension indiscernibility existence" lemma 1.7. Using stability, we have that for any sequence $\left\langle a_{\alpha}: \alpha<\lambda\right\rangle$ we get a subsequence $\left\langle a_{\alpha}: \alpha \in S\right\rangle, S \subseteq \lambda$ stationary such that the $\Delta$-type of $\left\langle a_{\alpha_{0}}, \ldots, a_{\alpha_{k}}\right\rangle$ over $\left\{a_{\beta}: \beta<\alpha_{0}\right\}$ does not depend on $\alpha_{k}$ when $\alpha_{0}<\ldots<\alpha_{k}$ are from $S$. We then improve it to "does not depend" on $\alpha_{k}, \alpha_{k-1}, \ldots, \alpha_{k-n}$ " for a fix $n$, but this does not give full indiscernibility. Note that the definition of stability and its use in 1.7 speak of definability of types but the type are model theoretic ones, speaking only on $\Delta$-formulas whereas the "definable" means in set theoretic sense, so the definition is arbitrary, just has to be in the submodel (of e.g. some $(\mathscr{H}(\chi), \in)$ ). This seems inherent in our 
framework: for a predicate $P \in \tau(\mathfrak{A})$, the set theoretic definition of $P^{\mathfrak{A}}$ may involve, as approximations, relations on the reals which are very complicated model theoretically.

In $\S 2$ we generalize the theorem "the order property implies unstability", in 2.1; giving a criterion for unstability in 2.4. Now the "unstable" in Definition 1.4(3) really speaks on having a perfect set of types; we here define apparently weaker version $(\mu, \Delta, \lambda)$-unstable.

Lastly, in $\S 3$, we define ranks, but here the ranks are for subsets $\mathbf{I}$ of ${ }^{m} \mathfrak{A}$, not just definable ones; as explained above this seems inherent in our framework.

We then prove (in $3.3,3.7)$ that " $\mathfrak{A}$ is $\left(\aleph_{0}, \Delta\right)$-stable" is really defined, i.e. that we have several equivalent definitions: some from the structure side, some from the non-structure side (generally on this see [She, $\S(1 \mathrm{~A}), \S(2 \mathrm{~A}), \S(2 \mathrm{~B})]$ ).

Lastly, we prove a theorem on the existence of (fully) indiscernible sub-sequence: a sequence of length $\lambda^{+\kappa^{+}}$has a sub-sequence of length $\lambda$; a parallel situation occurs for strongly dependent $T$ (by [She14]). We end noting that, of course, being $\left(\aleph_{0}, \Delta\right)$ unstable implies a failure of categoricity (strong one) as expected in our frame.

On the existence of indiscernibles in stable models (not necessarily of a stable theory) and history, see [She09c].

\section{$\S 0(\mathrm{C})$. Further Comments.}

Definition 0.10. 1) For a definition $\mathfrak{A}$ of a $\tau$-model (usually with a set of elements a definable set of reals) we say that $\mathfrak{A}$ is categorical 2 in $\lambda<2^{\aleph_{0}}$ when: for some $A \subseteq \lambda$ : for every $A_{1}, A_{2} \subseteq \lambda$ the models $\mathfrak{A}^{\mathbf{L}\left[A_{1}, A\right]}, \mathfrak{A}^{\mathbf{L}\left[A_{2}, A\right]}$ are isomorphic (in $\mathbf{V}$ ).

2) For a class $\mathfrak{K}$ of forcing notions and cardinal $\lambda$ we say $\mathfrak{A}$ is categorical ch $_{2}$ in $(\lambda, \mathfrak{K})$ when for every $\mathbb{P} \in \mathfrak{K}$ satisfying ${ }^{1} \Vdash_{\mathbb{P}}$ " $2^{\aleph_{0}}>\lambda$ ", we have in $\mathbf{V}^{\mathbb{P}}$ : the structure $\mathfrak{A}$ is categorical 2 in $\lambda$, i.e. in the sense of part (1).

3) Note the $\lambda$ may be a given cardinal or definition of one.

Comparing Definition 0.10(1) with the forcing version we lose when $\mathbf{V}=\mathbf{L}$, as it says nothing, we gain as (when $2^{\aleph_{0}}>\aleph_{1}$ ) we do not have to go outside the universe. Maybe best is categorical in $\lambda$ in $\mathbf{V}^{\mathbb{P}}$ for every c.c.c. forcing notion $\mathbb{P}$ making $2^{\aleph_{0}}>\lambda$, see $\S 4$. Of course, the examples from 0.8 works also here.

Note also that it may be advisable in $0.10(1)$ to restrict ourselves to the case $\lambda$ is regular as we certainly like to avoid the possibility $\left(2^{\aleph_{0}}\right)^{\mathbf{L}\left[A_{1}, r\right]}=\lambda<\left(2^{\aleph_{0}}\right)^{\mathbf{L}\left[A_{2}, r\right]}$ (see on this and for history in [She94, Ch.VII]).

Of course, any reasonably absolute definition of unstability implies non-categoricity: if we have many types we should have a perfect set of them, hence adding Cohen subsets of $\omega$ adds more types realized. If we add $\left\langle\eta_{i}: i<2^{\aleph_{0}}\right\rangle$ Cohen reals then $\left\langle\mathfrak{A}^{\mathbf{V}\left[\eta_{i}: i \in A\right]}: A \subseteq 2^{\aleph_{0}}\right\rangle$ is a sequence of pairwise non-isomorphic over the countable set of parameters, if we get $2^{2^{\aleph_{0}}}$ or just $>2^{\aleph_{0}}$ non-isomorphic models, we can forget the parameters and retain our "richness in models". The present work is to some extent a continuation of [She84], [She99a], see history there.

Remark 0.11. For $\ell(*)=1,2$ letting $\kappa=\aleph_{\ell(*)-1}$, we may replace everywhere $\kappa$ Suslin, $\kappa, \kappa^{+}$by $\Sigma_{\ell(*)}^{1}, \aleph_{\ell(*)-1}, \aleph_{\ell(*)}$ respectively. Presently, though the first (i.e.

\footnotetext{
${ }^{1}$ We may allow $\Vdash_{\mathbb{P}}$ " $2^{\aleph_{0}} \geq \lambda$ ", but then for some $\mathbb{P}_{1}$ there is a $\mathbb{P}_{2}$-name $\underset{\sim}{A} \subseteq \lambda$ such that if

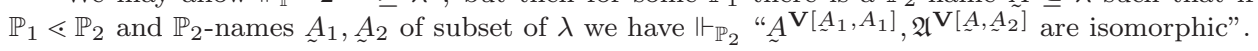


$\left.\Sigma_{1}^{1}\right)$ case is more restrictive than the second ( $\aleph_{1}$-Suslin) case, what we can prove is the same. For $\ell(*)=1$ we have real equivalence.

Note that below the indiscernible sequences are indiscernible sets, justified by 2.1 (see, e.g. [She90]).

Claim 0.12. 1) Assume $I$ is an infinite linear order $\bar{a}_{s} \in{ }^{m} \mathfrak{A}$ for $s \in I,\left\langle\bar{a}_{s}: r \in I\right\rangle$ is a $(\Delta, n)$-indiscernible sequence (see 1.9) but not a $(\Delta, n)$-indiscernible set (see 1.9(9)). Then we can find $\varphi=\varphi\left(\bar{x}_{0}, \ldots, \bar{x}_{n}, \bar{y}\right) \in \Delta$ and $\bar{b} \in{ }^{\ell g(\bar{y})} A$ and $\ell<n$ such that: if $s_{0}<_{I} \ldots<_{I} s_{n}$ then

(a) $M \models \varphi\left[\bar{a}_{s_{0}}, \ldots, \bar{a}_{s_{n}}, \bar{b}\right]$

(b) $M \models \varphi\left[\bar{a}_{t_{0}}, \ldots, \bar{a}_{t_{n}}, \bar{b}\right]$ when

- $t_{k}=s_{k}$ if $k \neq \ell, \ell+1$

- $t_{k}=s_{k+1}$ if $k=\ell$

- $t_{k}=s_{k-1}$ if $k=\ell+1$.

2) So if $I=\lambda$, this implies the $(\lambda, \Delta)$-order property.

3) Similarly for end-( $\Delta, n)$-indiscernible sequence (but we may have to demand $s_{0} \geq s_{*} \in I$ for a fix $s_{*}$.

Recall

Definition 0.13. 1) Let $\mathbf{T}_{\kappa}$ be the following tree:

(a) members have the form $t=\left(\nu_{t}, \eta_{t}\right)$ such that for some $\left.n, \eta_{t} \in{ }^{n} \omega, \nu_{t} \in{ }^{n} \kappa\right\}$

(b) it is ordered by $s \leq_{\mathbf{T}_{\kappa}} t$ iff $\eta_{s} \unlhd \eta_{t} \wedge \nu_{s} \unlhd \nu_{t}$.

2) For a subtree $\mathscr{T}$ of $\mathbf{T}_{\kappa}$ let $\operatorname{proj}(\mathscr{T})=\left\{\eta \in{ }^{\omega} \omega\right.$ : for some $\nu \in{ }^{\omega} \kappa$ we have $(\nu, \eta) \in \lim (\mathscr{T})\}$.

3) A set of this form is called $\kappa$-Suslin.

4) For notational convenience we may replace ${ }^{\omega} \omega$ by ${ }^{\omega}\left(\mathscr{H}\left(\aleph_{0}\right)\right)$ and consider $\bar{a} \in$ ${ }^{m}(\mathfrak{A})$ as $\left\langle\left\langle a_{\ell}(n): \ell<\ell g(\bar{a})\right\rangle: n<\omega\right\rangle$ and let $\bar{a} 1 n=\left\langle\left\langle a_{\ell}(k): \ell\langle\ell g(\bar{a})\rangle: k\langle n\rangle\right.\right.$; (similarly we may replace ${ }^{\omega} \kappa$ by ${ }^{\omega}\left(\mathbf{L}_{\kappa}\right)$, too). 


\section{$\S$ 1. Generalizing Stability IN $\aleph_{0}$}

We may consider the dividing line for abelian groups from [She99b] and try to generalize it for any simply defined (e.g. anywhere from a Polish algebra until $\kappa$-Suslin) model. We deal with trying to prove that we have two possibilities, in the high, complicated side we get a parallel of non $\aleph_{0}$-stability (hence strong non-categoricity, see 3.10); in the low side we have a rank. But even for minimal formulas, the example in [She11, §5] shows that we are far from being done, still we may be able to say something on the structure.

We may consider also ranks parallel to the ones for superstable theories. Recall that there are two kinds of definability we are considering: the model theoretic one and the set theoretic one.

Context 1.1. 1) If not said otherwise, $\mathfrak{A}$ will be a structure with countable vocabulary and its set of elements is a set of reals; usually a definition - see 0.5(1).

2) $\mathscr{L}$ is a logic. We did not specify the logic; we may assume it is $\subseteq \mathbb{L}_{\aleph_{1}, \aleph_{0}}$ or just $\mathbb{L}_{\aleph_{1}, \aleph_{0}}(\mathbf{Q})$ where $\mathbf{Q}$ is the quantifier "there are uncountably many".

3) $\kappa$ is a fix cardinality, let a $\kappa$-model mean a $\kappa$-Suslin model.

Definition 1.2. 1) For a structure $\mathfrak{A}$, an $\mathfrak{A}$-formula or ( $\mathfrak{A}, \mathscr{L}$ )-formula $\varphi$ is a formula in the language $\mathscr{L}\left(\tau_{\mathfrak{A}}\right)$ so in the logic $\mathscr{L}$, and the formula is in the vocabulary of $\mathfrak{A}$ with finitely many free variables, writing $\varphi=\varphi(\bar{x})$ means that $\bar{x}$ is a finite sequence of variables with no repetitions including the free variables of $\varphi$.

2) $\Delta$ denotes a set of such formulas and $\bar{\varphi}$ denotes a pair $\left(\varphi_{0}(\bar{x}), \varphi_{1}(\bar{x})\right)$ of formulas so $\bar{\varphi}$ is a $\Delta$-pair when $\varphi_{0}, \varphi_{1} \in \Delta$; in general we do not require any closure properties on $\Delta$.

3) We say $\varphi$ (or $\Delta$ or $\bar{\varphi}$ ) is $\kappa$-Suslin (or $\Sigma_{1}^{1}$ or $\Sigma_{2}^{1}$ or $\Delta_{0}^{1}(=$ Borel)) iff they are so as set theoretic formulas, see $1.3(2)$.

4) We say $\mathfrak{A}$ is a $\kappa$-model or a $\kappa$-Suslin model when being a member, (that is, in its universe) its relation and its functions are $\kappa$-Suslin; so the definition of $\mathfrak{A}$ is the sequence of $\kappa$-Suslin definitions and saying, e.g. $\mathfrak{A} \in N \subseteq(\mathscr{H}(\chi), \theta)$ means that also this sequence of $\kappa$-Suslin definitions belongs to $N$.

Discussion 1.3. 1) So if the relation " $\eta \in \mathfrak{A}$ ", " $\left(\eta_{0}, \ldots, \eta_{n-1}\right) \in P^{\mathfrak{A}}$ " where $P$ is an $n$-place predicate ${ }^{2}$ of $\tau_{\mathfrak{A}}$, are $\kappa$-Suslin, then they are upward and downward absolute (as long as the relevant subtrees of $\mathbf{T}_{\kappa}$ are in the universe).

2) When we consider a formula $\varphi(\bar{x})$ this is more complicated but we are assuming it, too, is $\kappa$-Suslin, meaning that this (set theoretic) definition defines $\varphi(\bar{x})^{\mathfrak{A}}$ also in the other universes we consider.

Definition 1.4. 1) We say $(\mathfrak{A}, \Delta$ ) is a $\kappa$-candidate (or $\kappa$-Suslin candidate; as $\kappa$ is constant we may omit it) when :

(a) $\mathfrak{A}$ is a $\kappa$-model

(b) $\Delta$ is a countable set of $(\mathfrak{A}, \mathscr{L})$-formulas ${ }^{3}$ which, are in the set theory sense, $\kappa$-Suslin (we identify $\varphi$ and $\neg \neg \varphi$ ), i.e. $\Delta$ contains the information saying which $\kappa$-Suslin relations are used

\footnotetext{
${ }^{2}$ pedantically we should change a little in 0.13 , or we can translate $P^{\mathfrak{A}} \subseteq{ }^{n}\left({ }^{\omega} \omega\right)$ to a subset of $\omega_{\omega}$

${ }^{3}$ We may use " $\Delta$ is a set of pairs $\bar{\varphi}=\left(\varphi_{0}(\bar{x}), \varphi_{1}(\bar{x})\right.$ of such formulas", and later demand $\bar{\varphi} \in \Delta$. So far it does not matter.
} 
(c) we consider changes of the universe (say by forcing) only when this is preserved, anyhow we can assume each $\varphi(\bar{x}) \in \Delta$ is quantifier free.

1A) We can replace being $\kappa$-Suslin by being $\Sigma_{1}^{1}$, by $\Sigma_{2}^{1}$, etc., (naturally we need enough absoluteness); if we replace it by $\Gamma$ we write $\Gamma$-candidate. If $\Gamma$ does not appear we mean it is $\kappa$-Suslin or understood from the context.

$2)$ If $(\mathfrak{A}, \Delta)$ is a candidate we say $\mathfrak{A}$ is $\left(\aleph_{0}, \Delta\right)$-stable (or $(\mathfrak{A}, \Delta)$ is $\aleph_{0}$-stable), when $\Delta$ is a countable set of $(\mathfrak{A}, \mathscr{L})$-formulas and for $\chi$ large enough and for every countable $N \prec\left(\mathscr{H}(\chi), \in,<_{\chi}^{*}\right)$ to which $(\mathfrak{A}, \Delta)$ belongs and $\bar{a} \in{ }^{m} \mathfrak{A}$ where $m<\omega$, the following weak definability condition on $\operatorname{tp}_{\Delta}(\bar{a}, N \cap \mathfrak{A}, \mathfrak{A})$ holds:

$\boxplus$ letting $\Phi_{\mathfrak{A}, \Delta}^{m}$ be defined in $(*)$, for some function $\mathbf{c} \in N$ with domain $\Phi_{(\mathfrak{A}, \Delta)}^{m}$ and range $\subseteq\{0,1\}$ the statement $(* *)$ holds where

$(*) \quad$ letting $\Phi_{\mathfrak{A}, \Delta}^{m}=\Phi_{(\mathfrak{A}, \Delta)}^{m}=\left\{\bar{\varphi}(\bar{x}, \bar{b}): \bar{\varphi}(\bar{x}, \bar{b})=\left(\varphi_{0}(\bar{x}, \bar{b}), \varphi_{1}(\bar{x}, \bar{b})\right)\right.$ and $\bar{x}=\left\langle x_{\ell}: \ell<m\right\rangle, \bar{b} \in \omega>\mathfrak{A}$ and $\varphi_{0}, \varphi_{1} \in \Delta$ and $\left.\mathfrak{A} \models \neg(\exists \bar{x}, \bar{y})\left(\varphi_{0}(\bar{x}, \bar{y}) \wedge \varphi_{1}(\bar{x}, \bar{y})\right)\right\}$,

$(* *) \quad$ if $\bar{\varphi}=\left(\varphi_{0}(\bar{x}, \bar{b}), \varphi_{1}(\bar{x}, \bar{b})\right) \in \Phi_{(\mathfrak{A}, \Delta)}^{m} \cap N$ and $\ell<2$ and $\mathfrak{A} \models \varphi_{\ell}[\bar{a}, \bar{b}]$ then $\ell=\mathbf{c}(\bar{\varphi})$.

$2 \mathrm{~A})$ We say $(\mathfrak{A}, \Delta)$ is $\mu$-stable when above we replace " $N$ countable" by " $N$ of cardinality $\mu$ and $\mu+1 \subseteq N^{\prime \prime}$.

3) We say that $(\mathfrak{A}, \Delta)$ is $\aleph_{0}$-unstable (or $\mathfrak{A}$ is $\left(\aleph_{0}, \Delta\right)$-unstable) when: there are $\bar{a}_{\eta} \in{ }^{m} \mathfrak{A}$ for $\eta \in{ }^{\omega} 2$ and $\varphi_{\nu, 0}\left(\bar{x}, \bar{y}_{\nu}\right) \in \Delta$ and $\varphi_{\nu, 1}\left(\bar{x}, \bar{y}_{\nu}\right) \in \Delta$ and $\bar{b}_{\nu} \in{ }^{\ell g(\bar{y})} \mathfrak{A}$ for $\nu \in{ }^{\omega>} 2$ such that:

(a) $\mathfrak{A} \models \neg(\exists \bar{x})\left(\varphi_{\nu, 0}\left(\bar{x}, \bar{b}_{\nu}\right) \wedge \varphi_{\nu, 1}\left(\bar{x}, \bar{b}_{\nu}\right)\right)$

(b) if $\nu \triangleleft \eta_{0}, \nu \triangleleft \eta_{1}$ and $\eta_{0}, \eta_{1} \in{ }^{\omega} 2, n=\ell g(\nu)$ and $\eta_{0}(n)=0, \eta_{1}(n)=1$ then $\mathfrak{A} \models \varphi_{\nu, 0}\left[\bar{a}_{\eta_{0}}, \bar{b}_{\nu}\right] \wedge \varphi_{\nu, 1}\left[\bar{a}_{\eta_{1}}, \bar{b}_{\nu}\right]$.

4) Let $\bar{\varphi}(\bar{x}, \bar{y}) \in \Phi_{(\mathfrak{A}, \Delta)}^{m}$ or " $\bar{\varphi}(\bar{x}, \bar{y})$ is a $\Delta$-pair" (pedantically ( $\left.\mathfrak{A}, \Delta\right)$-pair) means that $\bar{\varphi}(\bar{x}, \bar{y})=\left(\varphi_{0}(\bar{x}, \bar{y}), \varphi_{1}(\bar{x}, \bar{y})\right)$ and $\varphi_{0}(\bar{x}, \bar{y}), \varphi_{1}(\bar{x}, \bar{y})$ belongs to $\Delta$ and $\mathfrak{A} \models$ $\neg(\exists \bar{x}, \bar{y})\left(\varphi_{0}(\bar{x}, \bar{y}) \wedge \varphi_{1}(\bar{x}, \bar{y})\right)$.

5) Let $\bar{\varphi}(\bar{x}, \bar{y}) \in \Delta$ means that $\bar{\varphi}(\bar{x}, \bar{y})=\left(\varphi_{0}(\bar{x}, \bar{y}), \varphi_{1}(\bar{x}, \bar{y})\right)$ and $\varphi_{0}(\bar{x}, \bar{y}), \varphi_{1}(\bar{x}, \bar{y})$ belongs to $\Delta$.

Remark 1.5. 1) There are obvious absoluteness results (for $\bar{\varphi} \in \Phi_{(\mathfrak{A}, \Delta)}^{m},(\mathfrak{A}, \Delta)$ is $\aleph_{0}$-unstable and $\aleph_{0}$-stable).

2) On those notions (stable and unstable) being complimentary see Theorem 3.3.

3) We may consider in Definition 1.4(1) using countable models or models of cardinality $\kappa$, it is not clear to us what is better but see 1.6 below.

Observation 1.6. 1) If $\Delta$ is closed under negation then in Definition 1.4(2) we equivalently can replace $\boxplus$ by:

$\boxplus^{\prime}$ for some $\mathbf{c} \in N$ we have: if $\varphi(\bar{x}, \bar{y}) \in \Delta$ and $\bar{b} \in{ }^{\ell g(\bar{y})} \mathfrak{A}$ and $\bar{b} \in N \underline{\text { then }}$ $\mathfrak{A} \models \varphi(\bar{a}, \bar{b})$ iff $\mathbf{c}(\varphi(\bar{x}, \bar{b}))=1$.

2) In Definition 1.4(2) for all $\chi>2^{\aleph_{0}}$ the following variants of the definition are equivalent:

(a) the original one 
(b) as there but omitting "countable" and $<_{\chi}^{*}$, (a well ordering of $\mathscr{H}(\chi)$ )

(c) there is a club of countable $N \subseteq(\mathscr{H}(\chi), \in)$ which satisfies the conclusion (i.e. there $\mathbf{c} \in N$ such that ...)

(d) for each $\Delta$-pair $\bar{\varphi}(\bar{x}, \bar{y})$ there is a club $\mathscr{S} \subseteq[\mathscr{H}(\chi)]^{\aleph_{0}}$ such that for every $u \in \mathscr{S}$ letting $N_{u}=(\mathscr{H}(\chi), \in)\left\lceil A\right.$ we have: for some function $\mathbf{c} \in N_{A}$

$(\alpha) \operatorname{Dom}(\mathbf{c})=\left\{\bar{\varphi}(\bar{x}, \bar{b}): \bar{b} \in{ }^{\ell g(\bar{y})} \mathfrak{A}\right\}$, Range $(\mathbf{c}) \subseteq\{0,1\}$

( $\beta$ ) for every $\bar{b} \in N_{A} \cap{ }^{m} \mathfrak{A}$ and $\ell<2$ if $\mathfrak{A} \models \varphi_{\ell}[\bar{a}, \bar{b}]$ then $\ell=\mathbf{c}(\bar{\varphi}(\bar{x}, \bar{b})$.

(e) like clause (d) replacing "club $\mathscr{S}$ " by "stationary $\mathscr{S}$ ".

3) In Definition 1.4(2), the demand " $N$ is countable" can be omitted (and in 1.4(2A) can weaken $\|N\|=\mu$ to $\|N\| \geq \mu$ ).

Proof. Straight; e.g. $(a) \Rightarrow(b)$ let $N^{*} \prec\left(\mathscr{H}(\chi), \in,<_{\chi}^{*}\right)$ be uncountable and such that $\mathfrak{A}, \Delta \in N^{*}$. Now for every countable $N \prec N^{*}$ to which $(\mathfrak{A}, \Delta)$ belongs there is $\mathbf{c}_{N} \in N$ as mentioned in the definition 1.4. Hence by normality of the club filter on $\left[N^{*}\right]^{\aleph_{0}}$, the family of countable subsets of $N^{*}$, for some $\mathbf{c}^{*}$ the set $\mathscr{S}^{\prime}=\{N: N \prec$ $N^{*}$ is countable and $\left.\mathbf{c}_{N}=\mathbf{c}^{*}\right\}$ is a stationary subset of $\left[N^{*}\right]^{\aleph_{0}}$, so $\mathbf{c}^{*}$ can serve for $N^{*}$.

Claim 1.7. The End-Extention Indiscernibility existence lemma 1) Assume:

(a) $(\alpha) \quad \Delta$ is closed under negation or just

( $\beta) \quad$ if $\mathfrak{A} \models " \neg \varphi(\bar{a}, \bar{b})$ " and $\varphi(\bar{x}, \bar{y}) \in \Delta$ then for some $\bar{\varphi}(\bar{x}, \bar{y}) \in \Phi_{\mathfrak{A}, \Delta}^{\ell g(\bar{x})}$ we have $\varphi_{0}=\varphi$ and $\mathfrak{A} \models \varphi_{1}[\bar{a}, \bar{b}]$

(b) $\Delta$ is closed under permuting the (free) variables, $m<\omega$

(c) $(\mathfrak{A}, \Delta)$ is an $\aleph_{0}$-candidate which is $\aleph_{0}$-stable (or just $\mu$-stable, $\mu<\lambda$, see $1.4(2 A)$

(d) $\aleph_{0}<\lambda=\operatorname{cf}(\lambda)$ and $S \subseteq \lambda$ is stationary

(e) $\bar{a}_{\alpha} \in{ }^{m} \mathfrak{A}$ for $\alpha<\lambda$

(f) $A \subseteq \mathfrak{A}$ has cardinality $<\lambda$.

Then for some stationary $S^{\prime} \subseteq S$ the sequence $\left\langle\bar{a}_{\alpha}: \alpha \in S^{\prime}\right\rangle$ is a $\Delta$-end extension indiscernible sequence over $A$ in $\mathfrak{A}$ (see Definition 1.9(3),(4),(5) below).

2) Moreover for any pregiven $n<\omega$ we can find stationary $S^{\prime} \subseteq S$ such that $\left\langle\bar{a}_{\alpha}: \alpha \in S^{\prime}\right\rangle$ is $(\Delta, n)$-end extension indiscernible over $A$ in $\mathfrak{A}$.

3) In part (2) we can find a club $E$ of $\lambda$ and regressive function $f_{n}$ on $S \cap E$ for $n<\omega$ such that:

(i) if $\alpha, \beta \in S \cap E \underline{\text { then }} f_{n+1}(\alpha)=f_{n+1}(\beta) \Rightarrow f_{n}(\alpha)=f_{n}(\beta)$

(ii) if $n<\omega$ and $\gamma<\lambda$, then the sequence $\left\langle\bar{a}_{\alpha}: \alpha \in S \cap E, f_{n}(\alpha)=\gamma\right\rangle$ is $(\Delta, n)$-end extension indiscernible sequence over $A$

$(\text { ii })^{+}$moreover, if $n<\omega$ and $\beta, \gamma<\lambda$ then $\left\langle\bar{a}_{\alpha}: \alpha \in S \cap E \backslash \beta\right.$ and $\left.f_{n}(\alpha)=\gamma\right\rangle$ is $(\Delta, n)$-end extension indiscernible sequence over $\cup\left\{\bar{a}_{\gamma}: \gamma<\beta\right\} \cup A$.

Remark 1.8. 1) This is a "first round on indiscernibility".

2) The assumption " $\Delta$ is closed under negation" is quite strong.

3) Really we get indiscernible sets by 0.12 and 2.1 .

4) The claim and proof are similar to [She90, Ch.III,4.23,pg.120-1]. But before proving we define: 
Definition 1.9. 1) Let $(\mathfrak{A}, \Delta)$ be a candidate. We say "'A has $(\lambda, \Delta)$-order" when:

$(*)_{\lambda}$ for some $m(*)<\omega$ and $\bar{\varphi}(\bar{x}, \bar{y}) \in \Phi_{\mathfrak{Q}, \Delta}^{m(*)}$ with $\ell g(\bar{x})=\ell g(\bar{y})$, this formula linear orders some $\mathbf{I} \subseteq{ }^{m(*)} \mathfrak{A}$ of cardinality $\lambda$, see part (2) for definition.

2) We say that the formula $\bar{\varphi}(\bar{x}, \bar{y})$ linear orders $\mathbf{I} \subseteq{ }^{m(*)} \mathfrak{A}$ iff for some $\left\langle\bar{a}_{t}: t \in I\right\rangle$ we have:

(a) $\mathbf{I}=\left\{\bar{a}_{t}: t \in I\right\}$

(b) $I$ is a linear order

(c) $\bar{\varphi}=\left(\varphi_{0}(\bar{x}, \bar{y}), \varphi_{1}(\bar{x}, \bar{y})\right) \in \Phi_{(\mathfrak{A}, \Delta)}^{m}$

(d) if $s<_{I} t$ then $\mathfrak{A} \models \varphi_{0}\left[\bar{a}_{s}, \bar{a}_{t}\right] \wedge \varphi_{1}\left[\bar{a}_{t}, \bar{a}_{s}\right]$.

3) For a linear order $J$ (e.g. a set of ordinals), we say $\left\langle\bar{a}_{t}: t \in J\right\rangle$ is a $\Delta$-endextension indiscernible sequence (over $A$ ) iff for any $n<\omega$ and $t_{0}<_{J}<\ldots<_{J}$ $t_{n-1}<_{J} t_{n}$, the sequences $\bar{a}_{t_{0}}{ }^{\wedge} \ldots \bar{a}_{t_{n-2}}{ }^{\wedge} \bar{a}_{t_{n-1}}$ and $\bar{a}_{t_{0}}{ }^{\wedge} \ldots{ }^{\wedge} \bar{a}_{t_{n-2}}{ }^{\wedge} \bar{a}_{t_{n}}$ realize the same $^{4} \Delta$-type (over $A$ ) in $\mathfrak{A}$.

4) We say that $\left\langle\bar{a}_{t}: t \in J\right\rangle$ is $\left(\Delta, n_{0}, n_{1}\right)$-end-extension indiscernible sequence over $A$ in $\mathfrak{A}$ when:

(a) $J$ a linear order for some $m, \bar{a}_{t} \in{ }^{m} \mathfrak{A}, A \subseteq \mathfrak{A}$

(b) if $\left\langle r_{\ell}: \ell<n_{0}\right\rangle,\left\langle s_{\ell}: \ell<n_{1}\right\rangle,\left\langle t_{\ell}: \ell<n_{1}\right\rangle$ are $<_{J}$-increasing sequences and $n_{0}>0 \wedge n_{1}>0$ implies $r_{n_{0}-1}<_{J} s_{0}, r_{n_{0}-1}<J$ t $t_{0} \underline{\text { then }}$

$\bar{a}_{r_{0}}{ }^{\wedge} \ldots{ }^{\wedge} \bar{a}_{r_{n_{0}-1}}{ }^{\wedge} \bar{a}_{s_{0}}{ }^{\wedge} \ldots{ }^{\wedge} \bar{a}_{s_{n_{1}-1}}$ and $\bar{a}_{r_{0}}{ }^{\wedge} \ldots{ }^{\wedge} \bar{a}_{r_{n_{0}-1}}{ }^{\wedge} \bar{a}_{t_{0}}{ }^{\wedge} \ldots{ }^{\wedge} \bar{a}_{t_{n_{1}-1}}$ realizes the same $\Delta$-type over $A$ in $\mathfrak{A}$

(c) if $J$ has a last element we allow to decrease $n_{0}$ and/or $n_{1}$.

$5)$ If we omit $n_{0}$ this means for every $n_{0}$, (so " $\Delta$-end extension..." means $(\Delta, 1)$-end extension.

6) We say $\left\langle\bar{a}_{t}: t \in I\right\rangle$ is $(\Delta, n)$-indiscernible sequence over $A$ when it is an $(\Delta, 0, n)$ indiscernible sequence over $A$.

7) We say $\left\langle\bar{a}_{t}: t \in I\right\rangle$ is a $\Delta$-indiscernible sequence over $A$ when it is an $(\Delta, n)$ indiscernible sequence over $A$ for every $n$.

8) In (4) if we replace above "sequence" by "set"; this means that for every permutation $\pi$ of $n_{1}$ in clause (4), also $\bar{a}_{r_{0}}{ }^{\wedge} \ldots{ }^{\wedge} \bar{a}_{r_{n_{0}-1}}{ }^{\wedge} \bar{a}_{s_{\pi(0)}}, \ldots, \bar{a}_{s_{\pi\left(n_{1}-1\right)}}$ realizes the same $\Delta$-type over $A$ in $\mathfrak{A}$; similarly in clause (c).

9) Like (8) for (5), (6), (7).

Proof. Proof of 1.7

1) Let $\left\langle N_{\alpha}: \alpha<\lambda\right\rangle$ be an increasing continuous sequence of elementary submodels of $\left(\mathscr{H}(\chi), \in,<_{\chi}^{*}\right)$ to which $\mathfrak{A}$ belongs, such that $\left\|N_{\alpha}\right\|<\lambda, N_{\alpha} \cap \lambda \in \lambda$ and $\alpha \subseteq N_{\alpha}$ and $\left\langle\bar{a}_{\alpha}: \alpha<\lambda\right\rangle \in N_{0}$ (hence $\bar{a}_{\alpha} \in N_{\alpha+1}$ ). For each $\alpha \in S$, applying 1.6(3) to $N_{\alpha}, \bar{a}_{\alpha}$ we get $\mathbf{c}_{\alpha} \in N_{\alpha}$ as in Definition 1.4(2). So for some $\mathbf{c}^{*}$ and some stationary subsets of $S^{\prime} \subseteq S$ of $\lambda$ we have $\alpha \in S^{\prime} \Rightarrow \mathbf{c}_{\alpha}=\mathbf{c}^{*}$.

Now let $\beta_{1}<\beta_{2}$ be from the set $S^{\prime}$ and we shall prove that $p_{1} \subseteq p_{2}$ when we define $p_{\ell}=\operatorname{tp}_{\Delta}\left(\bar{a}_{\beta_{\ell}}, \cup\left\{\bar{a}_{\alpha}: \alpha<\beta_{\ell}\right\} \cup A, \mathfrak{A}\right)$ for $\ell=1,2$. So assume $\varphi=\varphi(\bar{x}, \bar{y}) \in$ $\Delta, \ell g(\bar{x})=m(*)$ and $\bar{b}$ is a sequence of length $\ell g(\bar{y})$ from $\cup\left\{\bar{a}_{\alpha}: \alpha<\beta_{1}\right\} \cup A$ and $\ell \in\{1,2\}$ and assume $\mathfrak{A} \models \neg \varphi\left[\bar{a}_{\beta_{\ell}}, \bar{b}\right]$ and it suffices to prove the $\mathfrak{A} \models \neg \varphi\left[\bar{a}_{\beta_{3-\ell}}, \bar{b}\right)$.

${ }^{4}$ We may consider "not containing a contradictory pair". 
Now there is $\bar{\varphi} \in \Phi_{\mathfrak{A}, \Delta}^{m(*)}$ such that $\varphi_{0} \equiv \varphi$ and $\mathfrak{A} \models \varphi_{1}\left[\bar{a}_{\beta_{\ell}}, \bar{b}\right]$. Why? Apply clause (a) of the assumption of our claim 1.7. If $(\alpha)$ there holds, that is, $\Delta$ is closed under negation choose $\bar{\varphi}(\bar{x}, \bar{y})=\left(\varphi_{0}(\bar{x}, \bar{y}), \neg \varphi_{0}(\bar{x}, \bar{y})\right)$, it is a $(\mathfrak{A}, \Delta)$-pair and necessarily $\mathfrak{A} \models \varphi_{1}\left[\bar{a}_{\beta_{\ell}}, \bar{b}\right]$; otherwise, that is sub-clause $(\beta)$ there holds so there is a $(\mathfrak{A}, \Delta)$-pair $\bar{\varphi}$ such that $\varphi_{0}=\varphi$ and $\mathfrak{A}=\varphi_{1}\left[\bar{a}_{\beta_{\ell}}, \bar{b}\right]$.

In both cases necessarily $\mathbf{c}_{\beta_{\ell}}\left(\bar{\varphi}\left(\bar{a}_{\beta_{\ell}}, \bar{b}\right)\right)=1$ but $\mathbf{c}_{\beta_{1}}=\mathbf{c}_{\beta_{2}}$ hence $\mathbf{c}_{\beta_{3-\ell}}\left(\bar{\varphi}\left(\bar{a}_{\beta_{3-\ell}}, \bar{b}\right)\right)=$ 1 which recalling $\boxplus(* *)$ in Definition 1.4 implies $\mathbf{c}_{\beta_{3-\ell}}\left(\bar{\varphi}\left(\bar{a}_{\beta_{3-\ell}}, \bar{b}\right) \neq 0\right.$ so by the choice of $\bar{\varphi}$ we have $\mathfrak{A} \models$ " $\neg \varphi_{0}\left[\bar{a}_{\beta_{3-\ell}}, \bar{b}\right]$ " which means $\mathfrak{A} \models " \neg \varphi\left[\bar{a}_{\beta_{3-\ell}}, \bar{b}\right]$ " as promised, so we are done.

2 ) We prove this by induction on $n$, that is, we prove by induction on $n$ that:

$\nabla_{\lambda}^{n}$ for all $m<\omega$, a stationary $S \subseteq \lambda$ and $\bar{a}_{\alpha} \in m_{\mathfrak{A}}$ for $\alpha<\lambda$ there is a stationary $S^{\prime} \subseteq S$ such that: if $\beta<\lambda, \alpha_{\ell}^{\prime} \in S^{\prime}, \alpha_{\ell}^{\prime \prime} \in S^{\prime}$ for $\ell<n$ and $\beta \leq$ $\alpha_{0}^{\prime}<\alpha_{1}^{\prime}<\ldots$ and $\beta \leq \alpha_{0}^{\prime \prime}<\alpha_{1}^{\prime \prime}<\ldots$ then $\bar{a}_{\alpha_{0}^{\prime}}{ }^{\wedge} \ldots \bar{a}_{\alpha_{n-1}^{\prime}}, \bar{a}_{\alpha_{0}^{\prime \prime}}{ }^{\wedge} \ldots{ }^{\wedge} \bar{a}_{\alpha_{n-1}^{\prime \prime}}$ realizes the same $\Delta$-type over $\cup\left\{\bar{a}_{\gamma}: \gamma<\beta\right\} \cup A$.

For $n=0$ the demand is empty so $S^{\prime}=S$ is as required. For $n=1$ apply the proof of part (1). For $n+1>1$, by the induction hypothesis we can find a stationary $S_{1} \subseteq S$ as required in $\otimes_{\lambda}^{n}$. For each $\alpha<\lambda$ we can choose $\beta_{\alpha, \ell}=\beta(\alpha, \ell)$ for $\ell \leq n$ such that $\alpha=\beta_{\alpha, 0}<\beta_{\alpha, 1}<\ldots<\beta_{\alpha, n}$ and $0<\ell \leq n \Rightarrow \beta_{\alpha, \ell} \in S_{1}$. Let $\bar{a}_{\alpha}^{*}=\bar{a}_{\beta_{\alpha, 0}}{ }^{\wedge} \cdots^{\wedge} \bar{a}_{\beta_{\alpha, n}}$ so $\bar{a}_{\alpha}^{*} \in{ }^{m(n+1)} \mathfrak{A}$ and apply the induction hypothesis or just the case $n=1$, i.e. part (1) to $m \times(n+1), S_{1},\left\langle\bar{a}_{\alpha}^{*}: \alpha<\lambda\right\rangle$ getting a stationary $S_{2} \subseteq S_{1}$ as required in $\otimes_{\lambda}^{n}$ or just in $\otimes_{\lambda}^{1}$.

Without loss of generality

$(*)_{1}$ for every $\gamma \in S_{2}$ and $\alpha \in \gamma$ we have $\beta_{\alpha, n}<\gamma$ hence

$(*)_{2}$ if $\gamma \in S_{2}$ then $\bigcup_{\alpha<\gamma} \bar{a}_{\alpha}^{*} \subseteq \bigcup_{\alpha<\gamma} \bar{a}_{\alpha}$, moreover, $\alpha<\gamma \wedge 0<\ell \leq n \Rightarrow \beta_{\alpha, \ell}<\gamma$.

We claim that $S_{2}$ is as required. So assume $\beta \leq \alpha_{0}^{\prime}<\ldots<\alpha_{n}^{\prime}<\lambda$ and $\beta \leq \alpha_{0}^{\prime \prime}<$ $\ldots<\alpha_{n}^{\prime \prime}<\lambda$ and $\alpha_{\ell}^{\prime}, \alpha_{\ell}^{\prime \prime} \in S_{2}$ for $\ell \leq n$.

Now, recalling $\beta(\alpha, \ell)=\beta_{\alpha, \ell}$ for $\ell \leq n, \alpha<\lambda$ we have:

(i) $\bar{a}_{\alpha_{0}^{\prime}}{ }^{\wedge} \bar{a}_{\alpha_{1}^{\prime}}{ }^{\wedge} \ldots{ }^{\wedge} \bar{a}_{\alpha_{n}^{\prime}}$ and $\bar{a}_{\alpha_{0}^{\prime}}{ }^{\wedge} \bar{a}_{\beta\left(\alpha_{0}^{\prime}, 1\right)}{ }^{\wedge} \ldots{ }^{\wedge} \bar{a}_{\beta\left(\alpha_{0}^{\prime}, n\right)}$ realizes the same $\Delta$-type over $\cup\left\{\bar{a}_{\gamma}: \gamma<\beta\right\} \cup A$ in $\mathfrak{A}$.

[Why? As $\ell \in\{1, \ldots, n\} \Rightarrow \beta\left(\alpha_{1}^{\prime}, \ell\right) \in S_{1} \wedge\left(\alpha_{\ell}^{\prime} \in S_{2} \subseteq S_{1}\right)$ and apply the choice of $S_{1}$ with $\alpha_{0}^{\prime}+1$ here standing for $\beta$ there.]

(ii) $\bar{a}_{\alpha_{0}^{\prime}} \bar{a}_{\beta\left(\alpha_{0}^{\prime}, 1\right)}{ }^{\wedge} \ldots{ }^{\wedge} \bar{a}_{\beta\left(\alpha_{0}^{\prime}, n\right)}$ is equal to $\bar{a}_{\alpha_{0}^{\prime}}^{*}$.

[Why? By the choice of $\bar{a}_{\alpha_{0}^{\prime}}^{*}$.]

(iii) $\bar{a}_{\alpha_{0}^{\prime}}^{*}, \bar{a}_{\alpha_{0}^{\prime \prime}}^{*}$ realizes the same $\Delta$-type over $\cup\left\{\bar{a}_{\gamma}^{*}: \gamma<\beta\right\} \cup A$ hence over $A \cup\left\{\bar{a}_{\gamma}: \gamma<\beta\right\}$.

[Why? By the choice of $S_{2}$ ].

Similarly

(iv) $\bar{a}_{\alpha_{0}^{\prime \prime}}^{*}$ is equal to $\bar{a}_{\alpha_{0}^{\prime \prime}} \bar{a}_{\beta\left(\alpha_{0}^{\prime \prime}, 1\right)}{ }^{\wedge} \ldots{ }^{\wedge} \bar{a}_{\beta\left(\alpha_{0}^{\prime \prime}, n\right)}$

(v) $\bar{a}_{\alpha_{0}^{\prime \prime}}{ }^{\wedge} \bar{a}_{\beta\left(\alpha_{0}^{\prime \prime}, 1\right)}{ }^{\wedge} \ldots{ }^{\wedge} \bar{a}_{\beta\left(\alpha_{0}^{\prime \prime}, n\right)}$ and $\bar{a}_{\alpha_{0}^{\prime \prime}}{ }^{\wedge} \bar{a}_{\alpha_{1}^{\prime \prime}}{ }^{\wedge} \ldots{ }^{\wedge} \bar{a}_{\alpha_{n}^{\prime \prime}}$ realizes the same $\Delta$-type over $\cup\left\{\bar{a}_{\gamma}: \gamma<\beta\right\} \cup A$. 
By (i)- (v) recalling $(*)_{2}$ the set $S_{2}$ is as required in $\bigotimes_{\lambda}^{n+1}$.

3) The proofs of parts (1), (2) actually give this.

Claim 1.10. In Theorem 1.7(3) we can add:

(iii) we have (where $e_{n}$ and $\left\langle S_{n, \gamma}: \gamma<\lambda\right\rangle$ are defined below)

$(\alpha)$ if $\gamma_{1}, \gamma_{2}<\lambda$ are $e_{n}$-equivalent and $S_{n+1, \gamma_{1}}, S_{n+1, \gamma_{0}}$ are stationary then for some $\Delta$-formula $\varphi\left(\bar{x}_{0}, \ldots, \bar{x}_{n}, \bar{y}\right)$ and sequence $\bar{b}$ of length $\overline{\ell g}(\bar{y})$ from $\cup\left\{\bar{a}_{\alpha}: \alpha<\min \left\{\gamma_{1}, \gamma_{2}\right\}\right\} \cup A$ we have:

- if $\alpha_{0}<\ldots<\alpha_{n}$ are from $S_{n+1, \gamma_{1}} \cup S_{n+1, \gamma_{2}}$ then $\mathfrak{A} \models \varphi\left[\bar{a}_{\alpha_{0}}, \ldots, \bar{a}_{\alpha_{n}}, \bar{b}\right]$ iff $\alpha_{0} \in S_{n+1, \gamma_{2}}$ where

$(\beta) e_{n}$ is the equivalent relation $\left\{\left(\gamma_{1}, \gamma_{2}\right)\right.$ : there are $\alpha_{1}>\gamma_{1}$ and $\alpha_{2}>\gamma_{2}$ (but $<\lambda$ ) such that $f_{n}\left(\alpha_{1}\right)=f_{n}\left(\alpha_{2}\right)$ but $f_{n+1}\left(\alpha_{1}\right)=\gamma_{1}, f_{n+1}\left(\alpha_{2}\right)=$ $\left.\gamma_{2}\right\}$

$(\gamma) S_{n, \gamma}=\left\{\alpha<\lambda: \alpha>\gamma\right.$ and $\left.f_{n}(\alpha)=\gamma\right\}$.

Proof. By induction on $n$. We choose $f_{n}^{\prime}$, a club $E_{n}^{\prime}$ and so $e_{n},\left\langle S_{n, \gamma}: \gamma<\lambda\right\rangle$ such that:

$\boxplus(a) \quad E_{n} \subseteq E$

(b) $E_{n}^{\prime},\left\langle f_{\ell}^{\prime}: \ell \leq n\right\rangle$ are as required in $1.7(3)$

(c) $S_{n, \gamma}$ are defined as in $($ iii $)(\gamma)$ above from $f_{n}^{\prime}$

(d) $e_{\ell}$ for $\ell<n$ is defined from $f_{\ell}^{\prime}, f_{\ell+1}^{\prime}$ as in $(\beta)$ above.

There are no problems. 


\section{$\S 2$. ORDER AND UnSTABILITY}

Lemma 2.1. The order/unstability lemma:

Assume that

$\nabla_{1}(a)(\mathfrak{A}, \Delta)$ is a $\kappa$-candidate, (e.g. $\ell(*) \in\{1,2\}, \kappa=\aleph_{\ell(*)-1}$ the relation are $\Sigma_{\ell(*)}^{1}$ hence a $\kappa$-Suslin)

(b) $\varphi_{0}(\bar{x}, \bar{y}), \varphi_{1}(\bar{x}, \bar{y}) \in \Delta$ are contradictory in $\mathfrak{A}$

(c) $J$ is a linear order of cardinality $\lambda$

(d) $\bar{a}_{t} \in{ }^{m} \mathfrak{A}$ for $t \in J$

(e) $\mathfrak{A} \models \varphi_{0}\left[\bar{a}_{s}, \bar{a}_{t}\right]$ and $\mathfrak{A} \models \varphi_{1}\left[\bar{a}_{t}, \bar{a}_{s}\right]$ whenever $s<J$

$\nabla_{2}\left(\right.$ a) $\quad \lambda \geq \aleph_{\kappa^{+}} \underline{\text { or }}$

(b) $J$ is with density $\mu<|J|$ and $\kappa<|J|$.

$\underline{\text { Then }}(\mathfrak{A}, \Delta)$ is $\aleph_{0}$-unstable; even more specifically the demand in Definition 1.4(3) holds with $\varphi_{\nu, 0}=\varphi_{0}, \varphi_{\nu, 1}=\varphi_{1}$.

Question 2.2. What can $\left\{\lambda: \mathfrak{A}\right.$ has a $(\Delta, \lambda)$-order, that is $\boxplus_{1}$ of 2.1 holds for $\left.J=\lambda\right\}$ be?

Remark 2.3. 1) Note that we cannot hope for too much (for 2.2), because a specific case is: $\lambda\}$.

$\{\lambda$ : in the Boolean algebra $\mathscr{P}(\mathbb{N}) /$ finite there is an increasing chain of length

2) However, if we replace $J \cong \lambda$ by $|J|=\lambda$, then we have something to say.

We first prove a claim from which we can derive the lemma.

Claim 2.4. If $\boxplus$ below holds, then the pair $(\mathfrak{A}, \Delta)$ is $\aleph_{0}$-unstable; moreover upward absolutely so (and downward if $(\mathfrak{A}, \Delta)$ are in the submodel) where:

$\boxplus(a) \quad(\mathfrak{A}, \Delta)$ is a $\kappa$-candidate and $m<\omega, \Phi=\Phi_{(\mathfrak{A}, \Delta)}^{m}$ or just $\Phi \subseteq \Phi_{(\mathfrak{A}, \Delta)}^{m}$

(b) $\overline{\mathscr{P}}=\left\langle\mathscr{P}_{\alpha}: \alpha<\kappa^{+}\right\rangle$

(c) $\mathscr{P}_{\alpha}$ is a non-empty family of subsets of ${ }^{m} \mathfrak{A}$

(d) if $\alpha<\beta<\kappa^{+}$and $\mathbf{I} \in \mathscr{P}_{\beta}$ then for some $\mathbf{I}_{0}, \mathbf{I}_{1} \in \mathscr{P}_{\alpha}$ which are subsets of $\mathbf{I}$ and some pair $\left(\varphi_{0}(\bar{x}, \bar{b}), \varphi_{1}(\bar{x}, \bar{b})\right) \in \Phi$ we have $\ell<2$ and $\bar{a} \in \mathbf{I}_{\ell} \Rightarrow \mathfrak{A} \models \varphi_{\ell}(\bar{a}, \bar{b})$

(e) if $\mathbf{I} \in \mathscr{P}_{\beta}$ and $\alpha<\beta<\kappa^{+}$and $F$ is a function with domain $\mathbf{I}$ and range of cardinality $\leq \kappa$, then there is $\mathbf{I}^{\prime} \in \mathscr{P}_{\alpha}$ such that $\mathbf{I}^{\prime} \subseteq \mathbf{I}$ and $F\left\lceil\mathbf{I}^{\prime}\right.$ is constant.

Proof. Proof of 2.1 from 2.4

Case 1: $\lambda \geq \aleph_{\kappa^{+}}$

For $\alpha<\kappa^{+}$we let

$\mathscr{P}_{\alpha}=\left\{\mathbf{I}: \mathbf{I} \subseteq{ }^{m} \mathfrak{A}\right.$ is linearly ordered by $\bar{\varphi}$ and has cardinality $\left.\geq \kappa^{+\alpha}\right\}$.

Case 2: $J$ has density $\mu,|J|>\mu+\kappa$ 
For $\alpha<\kappa^{+}$we let

$$
\begin{aligned}
\mathscr{P}_{\alpha}=\{\mathbf{I}: \quad & \mathbf{I} \subseteq{ }^{m} \mathfrak{A} \text { is linearly ordered by } \bar{\varphi} \text { getting an order of cardinality } \\
& >\mu+\kappa \text { and density } \leq \mu\} .
\end{aligned}
$$

This should be clear.

By $\nabla_{2}$ of the assumption, at least one of the cases holds.

Proof. Proof of 2.4

For each $\varphi(\bar{x}) \in \Delta$ as $\left\{\bar{a} \in{ }^{\ell(\bar{x})} \mathfrak{A}: \mathfrak{A} \models \varphi[\bar{a}]\right\}$ is a $\kappa$-Suslin set and $^{5}$

$(*)_{\varphi(\bar{x})}$ we can find $\left\langle\mathscr{T}_{\varphi}: \varphi \in \Delta\right\rangle$ such that (recalling 0.13(4))

(a) if $\alpha<\kappa$ then $\mathscr{T}_{\varphi}$ is a closed subtree of $\mathbf{T}_{\kappa}$, see 0.13

(b) $\{\bar{a} \in \ell g(\bar{x}) \mathfrak{A}: \mathfrak{A} \models \varphi[\bar{a}]\}=\left\{\bar{a}\right.$ : some $\nu \in{ }^{\omega} \kappa$ witness $\varphi[\bar{a}]$, that is $\left.(\bar{a}, \nu) \in \lim \left(\mathscr{T}_{\varphi}\right)\right\}$.

We can find functions $F_{\varphi}$ such that if $\varphi(\bar{x}) \in \Delta$ and $\mathfrak{A} \models \varphi[\bar{a}]$ then $F_{\varphi}(\bar{a}) \in{ }^{\omega} \kappa$ witnesses this. For notational simplicity and without loss of generality $m=1$. Let $W=\left\{w: w \subseteq{ }^{\omega>} 2\right.$ is a front ${ }^{6}$ hence finite $\}$.

For $w \in W$ and $n<\omega$ let $Q_{n, w}$ be the family of objects $\mathfrak{x}=(w, n, \bar{u}, \bar{\nu}, \bar{\varrho}, \bar{\varphi})=$ $\left(w_{\mathfrak{x}}, n_{\mathfrak{x}}, \ldots\right)$ such that:

$(*)_{\mathfrak{x}}$ for unboundedly many $\alpha<\kappa^{+}$we can find a witness (or an $\alpha$-witness) $\mathbf{y}=\left(\left\langle\bar{a}_{\ell}: \ell<n\right\rangle,\left\langle B_{\rho}: \rho \in w\right\rangle\right)$ for $\mathfrak{x}$ which means:

(a) $(\alpha) \quad \bar{u}=\left\langle\left(u_{\rho}^{0}, u_{\rho}^{1}\right): \rho \in w\right\rangle$

$(\beta) \quad$ if $\rho \in w$ then $u_{\rho}^{0}, u_{\rho}^{1} \subseteq n$ are disjoint

$(\gamma) \quad \bar{\varphi}=\left\langle\bar{\varphi}^{\ell}: \ell<n\right\rangle$ where $\bar{\varphi}^{\ell}=\left(\varphi_{0}^{\ell}\left(\bar{x}, \bar{y}_{\ell}\right), \varphi_{1}^{\ell}\left(\bar{x}, \bar{y}_{\ell}\right)\right) \in \Phi_{(\mathfrak{A}, \Delta)}^{m}$

(b) $\bar{a}_{\ell} \in{ }^{\ell g\left(\bar{y}_{\ell}\right)} \mathfrak{A}$

(c) $B_{\rho} \in \mathscr{P}_{\alpha}$ for $\rho \in w$

(d) if $\rho \in w, \bar{b} \in B_{\rho}$ and $\ell<n$ then $\left(\varphi_{0}^{\ell}\left(\bar{x}, \bar{a}_{\ell}\right), \varphi_{1}^{\ell}\left(\bar{x}, \bar{a}_{\ell}\right)\right) \in \Phi_{(\mathfrak{A}, \Delta)}^{m}, \ell g(\bar{x})=$ $m, \ell g\left(\bar{y}_{\ell}\right)$ arbitrary (but finite) and

( $\alpha) \quad \ell \in u_{\rho}^{0} \Rightarrow \mathfrak{A} \models \varphi_{0}^{\ell}\left[\bar{b}, \bar{a}_{\ell}\right]$

( $\beta) \quad \ell \in u_{\rho}^{1} \Rightarrow \mathfrak{A} \models \varphi_{1}^{\ell}\left[\bar{b}, \bar{a}_{\ell}\right]$

(e) if $\nu \neq \rho$ are from $w$ then $\left(u_{\rho}^{0} \cap u_{\nu}^{1} \neq \emptyset\right) \vee\left(u_{\nu}^{0} \cap u_{\rho}^{1} \neq \emptyset\right)$

(f) $(\alpha) \quad \bar{\nu}=\left\langle\nu_{\rho, \ell}^{i}: \rho \in w, i \in\{0,1\}\right.$ and $\left.\ell \in u_{\rho}^{i}\right\rangle$

(B) $\bar{\varrho}=\left\langle\varrho_{\rho, \ell}^{i}: \rho \in w, i \in\{0,1\}\right.$ and $\left.\ell \in u_{\rho}^{i}\right\rangle$

$(\gamma) \quad \nu_{\rho, \ell}^{i} \in \omega>\omega$ pedantically $\in{ }^{\omega>} \mathscr{H}\left(\aleph_{0}\right)$

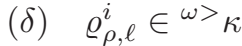

(g) if $\rho \in w, \bar{b} \in B_{\rho}, i \in\{0,1\}, \ell \in u_{\rho}^{i}$ then $\varrho_{\rho, \ell}^{i} \triangleleft F_{\varphi_{i}^{\ell}}^{0}\left(\bar{b}, \bar{a}_{\ell}\right)$ and $\nu_{\rho, \ell}^{i} \triangleleft\left(\bar{b}^{\wedge} \bar{a}_{\ell}\right)$, see $0.13(4)$.

Clearly

$\boxplus_{1} Q_{0,\{<>\}} \neq \emptyset$.

${ }^{5}$ Pedantically note that we sometimes consider $\bar{a} \in \ell g(\bar{x}) \mathfrak{A}$ as a member of $\omega_{\omega}$ or better $\omega\left(\mathscr{H}\left(\aleph_{0}\right)\right)$ rather than ${ }^{\ell g(\bar{x})}\left({ }^{\omega} \omega\right)$ or see $0.13(4)$.

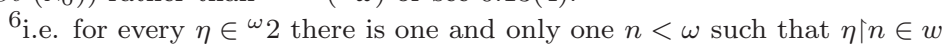


[Why? Let $\mathfrak{x}$ be such that $w_{\mathfrak{x}}=\{\langle\rangle\}, n_{\mathfrak{x}}=0, \bar{u}_{\mathfrak{x}}=\langle\rangle, \bar{\varrho}_{\mathfrak{x}}=\langle\rangle, \bar{\nu}_{\mathbf{x}}=\langle\rangle$ and $\bar{\varphi}_{\mathfrak{x}}=\langle\rangle$ and if $\alpha<\kappa^{+}$choose $\mathbf{I} \in \mathscr{P}_{\alpha}$ we let $B_{<>}=\mathbf{I}$; recall that by clause (c) of Claim 2.4 the family $\mathscr{P}_{\alpha}$ is non-empty.]

Next (the aim is to increase the length of the $\nu$ 's (so $\mathfrak{y}=\mathfrak{x}$ cannot work)

$\boxplus_{2}$ if $\mathfrak{x} \in Q_{n, w}$ and $\rho \in w \underline{\text { then }}$ there is $\mathfrak{y}$ such that:

- $\mathfrak{y} \in Q_{n, w}$

- $\bar{u}_{\mathfrak{y}}=\bar{u}_{\mathfrak{x}}$

- $\nu_{\mathfrak{x}, \rho, \ell}^{i} \triangleleft \nu_{\mathfrak{y}, \rho, \ell}^{i}$

- $\varrho_{\mathfrak{y}, \rho, \ell}^{i} \triangleleft \varrho_{\mathfrak{x}, \rho, \ell}^{i}$

- $\bar{\varphi}_{\mathfrak{y}}=\bar{\varphi}_{\mathfrak{x}}$.

[Why? As $\mathfrak{x} \in Q_{n, w}$ we know that for some unbounded $Y \subseteq \kappa^{+}$for each $\alpha \in Y$ there is an $\alpha$-witness $\mathbf{y}_{\alpha}=\left(\left\langle\bar{a}_{\ell}^{\alpha}: \ell<n\right\rangle,\left\langle B_{\rho}^{\alpha}: \rho \in w\right\rangle\right)$ as required in $(*)_{\mathfrak{x}}$. Let $\alpha<\kappa^{+}$and $\beta(\alpha)=\operatorname{Min}(Y \backslash(\alpha+1))$. Now for each $\rho \in w$ we have $B_{\rho}^{\beta(\alpha)} \in \mathscr{P}_{\beta(\alpha)}$.

Let $k>\sup \left\{\ell g\left(\nu_{\mathfrak{x}, \rho, \ell}^{i}\right), \ell g\left(\varrho_{\mathfrak{x}, \rho, \ell}^{i}\right): i<2, \rho \in w\right.$ and $\left.\ell<n\right\}+1$ and, of course, the set $\left\{F_{\varphi_{\mathrm{x}, \ell}^{\iota}}\left(\bar{b}, \bar{a}_{\ell}^{\beta(\alpha)}\right)\left\lceil k: \bar{b} \in B_{\rho}^{\beta(\alpha)}\right\}\right.$ has cardinality $\leq \kappa$ for each $i<2, \iota<2, \rho \in$ $w, \ell \in u_{\mathfrak{x}, \rho}^{i}$. Hence by clause (e) of Claim 2.4 recalling $\alpha<\beta(\alpha)$ we can find a subset $B_{\alpha, \rho}$ of $B_{\rho}^{\beta(\alpha)}$ from $\mathscr{P}_{\alpha}$ and $\nu_{\rho, \ell}^{\alpha, i} \in{ }^{\omega>} \mathscr{H}\left(\aleph_{0}\right), \varrho_{\rho, \ell}^{\alpha, i} \in{ }^{\omega>} \kappa$ such that

- $\bar{b} \in B_{\alpha, \rho} \Rightarrow\left(\bar{b}^{\wedge} \bar{a}_{\mathfrak{x}, \ell}\right) 1 k=\nu_{\rho, \ell}^{\iota}$

- $\bar{b} \in B_{\alpha, \rho} \Rightarrow F_{\varphi_{\mathfrak{x}, \ell}^{\ell}}\left(\bar{b}, \bar{a}_{\mathfrak{x}, \ell}\right)\left\lceil k=\varrho_{\rho, \ell}^{\alpha, i}\right.$.

Similarly for some $\left\langle\nu_{\rho, \ell}^{*, i}, \varrho_{\rho, \ell}^{*, i}: i<2, \rho \in w, \ell \in u_{\mathfrak{x}, \rho}^{i}\right\rangle$ we have

- $Y^{\prime}=\left\{\alpha \in Y: \nu_{\rho, \ell}^{\alpha, i}=\nu_{\rho, \ell}^{*, \iota}\right.$ and $\varrho_{\rho, \ell}^{\alpha, i}=\rho_{\rho, \ell}^{*, i}$ for $\rho \in w, \iota \in\{0,1\}$ end $\left.\ell \in u_{\rho}^{i}\right\}$ is unbounded in $\kappa^{+}$.

Now it is easy to choose $\mathfrak{y}$.]

$\boxplus_{3}$ if $\mathfrak{x} \in Q_{n, w}$ and $\rho \in w$ and $v=(w \backslash\{\rho\}) \cup\left\{\rho^{\wedge}\langle 0\rangle, \rho^{\wedge}\langle 1\rangle\right\}$ so $v \in W$, then there is $\mathbf{y} \in Q_{n+1, v}$ such that:

$(\alpha) \bullet n_{\mathbf{y}}=n_{\mathfrak{x}}+1$

$(\beta) \bullet u_{\mathbf{y}, \eta}^{i}=u_{\mathfrak{x}, \eta}^{i}$ for $\eta \in w \backslash\{\rho\}, i=0,1$

- $u_{\mathbf{y}, \rho^{\wedge}<j>}^{i} \cap\{0, \ldots, n-1\}=u_{\mathfrak{x}, \rho}^{i}$ for $i=0,1$ and $j=0,1$

- $n \in u_{\mathbf{y}, \rho^{\wedge}<j>}^{i} \Leftrightarrow j=i$ for $i, j \in\{0,1\}$

$(\gamma) \bullet \nu_{\mathbf{y}, \eta, \ell}^{i}=\nu_{\mathfrak{x}, \eta, \ell}^{i}$ for $\eta \in w \backslash\{\rho\}$

- $\nu_{\mathbf{y}, \rho^{\wedge}<\iota>, \ell}^{i}=\nu_{\mathfrak{x}, \rho, \ell}^{i}$

$(\delta) \bullet \varrho_{\mathbf{y}, \eta, \ell}^{i}=\varrho_{\mathfrak{x}, \eta, \ell}^{i}$ for $\eta \in w \backslash\{\rho\}$ and $\ell<m$

- $\varrho_{\mathbf{y}, \rho^{\wedge}<\iota>, \ell}^{i}=\varrho_{\mathfrak{x}, \rho, \ell}^{i}$ for $\ell<n$

- $\varrho_{\mathbf{y}, \rho^{\wedge}<\iota>, \ell}^{i}=\langle\rangle$ for $i=\iota, \ell=n_{\mathfrak{x}}$

(ع) $\bar{\varphi}_{\mathfrak{y}} \mid n=\bar{\varphi}_{\mathfrak{x}}$. 
[Why? Now we use clause (d) of the assumption of Claim 2.4 but we shall elaborate. Choose $Y$ and $\mathbf{y}_{\alpha}=\left(\left\langle a_{\ell}^{\alpha}: \ell<n\right\rangle,\left\langle B_{\rho}^{\alpha}: \rho \in w\right\rangle\right)$ for $\alpha \in Y$ as in the proof of $\boxplus_{2}$. For each $\alpha<\kappa^{+}$let $\left.\beta(\alpha)=\min (Y \backslash(\alpha+1))\right)$ and we apply clause (d) of $\oplus$ of 2.4 with $\alpha, \beta(\alpha), B_{\rho}^{\beta(\alpha)}$ here standing for $\alpha, \beta$, I there.

So there are $\bar{\varphi}(\bar{x}, \bar{c}), \mathbf{I}_{0}, \mathbf{I}_{1}$ such that:

$$
\begin{aligned}
\boxplus_{3.1} & \text { (a) } \bar{\varphi}(\bar{x}, \bar{c}) \in \Phi_{(\mathfrak{A}, \Delta)}^{m} \\
& \text { (b) } \mathbf{I}_{0}, \mathbf{I}_{1} \subseteq B_{\rho}^{\beta(\alpha)} \\
& \text { (c) } \mathbf{I}_{0}, \mathbf{I}_{1} \in \mathscr{P}_{\alpha} \\
& \text { (d) } \text { if } j<2 \text { and } \bar{a} \in \mathbf{I}_{j} \text { then } \mathfrak{A} \models \varphi_{j}[\bar{a}, \bar{c}] .
\end{aligned}
$$

Now $\mathfrak{y}$ is almost defined in $\boxplus_{3}$, the missing points are:

- we choose $\bar{\varphi}_{\mathfrak{y}, n}\left(\bar{x}, \bar{y}_{n}\right)$ as $\bar{\varphi}$

- we choose $\bar{a}_{\mathfrak{y}, n}$ as $\bar{c}$.

Now it suffices for every $\alpha<\kappa$ to choose an $\alpha$-witness $\mathbf{y}_{\alpha}^{\prime}$ of $\mathfrak{y}$, it is defined by

- $\bar{a}_{\ell}=a_{\ell}^{\beta *(\alpha)}$ for $\ell<n$

- $\bar{a}_{\ell}=\bar{c}$ for $\ell<n$

- $B_{\eta}$ is $B_{\eta}^{\beta(\alpha)}$ if $\eta \in \omega \backslash\{\rho\}$ and is $\mathbf{I}_{j}$ if $\eta=\rho^{\wedge}\langle j\rangle, j<2$.

Now check that $\mathfrak{y}$ is as required in $\boxplus_{3}$.]

$\boxplus_{4}$ we can choose $w_{n}, \rho_{n}$ by induction on $n$ such that

(a) $w_{n}$ is a front of ${ }^{n>} 2$, included in ${ }^{k} 2 \cup^{k+1} 2$ for some $k=k(n)$

(b) $w_{0}=\{\langle\rangle\}$

(c) $w_{n+1}=\left(w_{n} \backslash\left\{\rho_{n}\right\}\right) \cup\left\{\rho_{n}{ }^{\wedge}\langle 0\rangle, \rho_{n}{ }^{\wedge}\langle 1\rangle\right\}$ for some $\rho_{n}$

(d) if $w_{n} \cap^{k(n)} 2 \neq \emptyset$ then $\rho_{n} \in w_{n} \cap^{k(n)} 2$ and $k(n+1)=k(n)$

(e) if $w_{n} \cap{ }^{k(n)} 2=\emptyset$ then $\rho_{n} \in w_{n}={ }^{k(n)+1} 2$ and $k(n+1)=k(n)+1$

(f) necessarily $\left\{\rho_{n}: n<\omega\right\}=\omega>2$.

[Why? Trivially.]

$\boxplus_{5}$ we can choose $\mathfrak{x}_{n}^{1}, \mathfrak{x}_{n}^{2}$ by induction on $n$ such that

(a) $\mathfrak{x}_{n}^{1}, \mathfrak{x}_{n}^{2} \in Q_{n, w_{n}}$

(b) $\mathfrak{x}_{n}^{2}$ is gotten from $\mathfrak{x}_{n}^{1}$ by $\boxplus_{2}$

(c) $\mathfrak{x}_{n+1}^{1}$ is gotten from $\mathfrak{x}_{n}^{2}$ by $\boxplus_{3}$ for $\rho_{n}$.

[Why? For $n=0$, choose $\mathfrak{x}_{n}^{1}$ by $\boxplus_{1}$ and then $\mathfrak{x}_{n}^{2}$ by $\boxplus_{2}$. For $n=k+1$ choose $\mathfrak{x}_{n}^{1}$ satisfying (c) by $\boxplus_{3}$ for $\rho_{n}$ and then choose $\mathfrak{x}_{n}^{2}$ satisfying (b) by $\boxplus_{2}$.]

$\boxplus_{6}$ we can choose continuous functions $\rho \mapsto \bar{a}_{\rho}$ and $\rho \mapsto \varrho_{\rho, n}$ for $n<\omega$ where $\rho$ varies on ${ }^{\omega} 2$ such that:

(a) $\bar{a}_{\rho} \in{ }^{m} \mathfrak{A}$

(b) $\varrho_{\rho, \ell} \in{ }^{\omega} \kappa$

(c) if $\rho_{1} \neq \rho_{2} \in \omega_{2}$ and we let $\rho=\rho_{1} \cap \rho_{2}, k=\ell g(\rho)$ and $n$ is such that $\rho_{n}=\rho$ then letting $\iota(\ell)=\rho_{\ell}(k)$ so $\iota(1) \neq \iota(2)$ are $<2$, then for $\ell=1,2$ we have: 
- $\varrho_{\rho_{\ell}, n}$ witness $\mathfrak{A} \models \varphi_{\mathfrak{x}_{n+1}, n}^{\ell(\ell)}\left(\bar{a}_{\rho}, \bar{c}_{\mathfrak{x}_{n+1}, n}\right)$.

[Why? See the $(*)_{\mathfrak{x}_{n+1}, \ell}$ and the choice of the $\mathfrak{x}$ 's.]

Clearly $\boxplus_{6}$ gives the desired conclusion (even absolutely).

Remark 2.5. This claim can be generalized replacing $\aleph_{0}$ by $\mu$, strong limit singular of cofinality $\aleph_{0}$.

Definition 2.6. Let $(\mathfrak{A}, \Delta)$ be a $\kappa$-candidate and $m<\omega$.

1) For $A \subseteq \mathfrak{A}$ and $\bar{a} \in{ }^{n} \mathfrak{A}$ let $\operatorname{tp}_{\Delta}(\bar{a}, A, \mathfrak{A})=\left\{\varphi(\bar{x}, \bar{b}): \varphi(\bar{x}, \bar{y}) \in \Delta\right.$ and $\bar{b} \in{ }^{\ell g(\bar{y})}(A)$ and $\mathfrak{A}=\varphi[\bar{a}, \bar{b}]\}$.

2) $\Phi_{\mathfrak{A}, \Delta, A}^{\mathrm{pr}, m}=\left\{\left(\varphi_{0}(\bar{x}, \bar{b}), \varphi_{1}(\bar{x}, \bar{b})\right): \varphi_{0}(\bar{x}, \bar{y}), \varphi_{1}(\bar{x}, \bar{y})\right.$ belong to $\Delta$ and $\bar{b} \in{ }^{\ell g(\bar{y})} A$ and $\bar{x}=\left\langle x_{\ell}: \ell<m\right\rangle$ and $\left.\mathfrak{A} \models \neg(\exists \bar{x})\left[\varphi_{0}(\bar{x}, \bar{b}) \wedge \varphi_{1}(\bar{x}, \bar{b})\right]\right\}$ where $A \subseteq \mathfrak{A}, \Delta$ a set of $\mathfrak{A}$-formulas, and so $\Phi_{\mathfrak{A}, \Delta}^{m}=\Phi_{\mathfrak{A}, \Delta, A}^{\mathrm{pr}, m}$ when $A$ is the universe of $\mathfrak{A}$.

3) $\mathbf{S}_{\Delta}^{m}(A, \mathfrak{A})=\left\{\operatorname{tp}_{\Delta}(\bar{a}, A, \mathfrak{A}): \bar{a} \in{ }^{m} \mathfrak{A}\right\}$ where $A \subseteq \mathfrak{A}$ and $\Delta$ a set of $\mathbb{L}\left(\tau_{\mathfrak{A}}\right)$-formulas.

Definition 2.7. 1) We say $(\mathfrak{A}, \Delta)$ is $(\mu, \Delta, \lambda)$-unstable when there are $M \subseteq \mathfrak{A}, m<$ $\omega$ and $\left\langle\bar{a}_{\alpha}: \alpha<\lambda\right\rangle$ such that:

(a) $\bar{a}_{\alpha} \in{ }^{m} \mathfrak{A}$

(b) if $\alpha \neq \beta$ are $<\lambda$ then for some $\left(\varphi_{0}(\bar{x}, \bar{b}), \varphi_{1}(\bar{x}, \bar{b})\right) \in \Phi_{\mathfrak{A}, \Delta, M}^{m}$ (see Definition $2.6(2))$ we have $\varphi_{0}(\bar{x}, \bar{b}) \in \operatorname{tp}_{\Delta}\left(\bar{a}_{\alpha}, M, \mathfrak{A}\right)$ and $\varphi_{1}(\bar{x}, \bar{b}) \in \operatorname{tp}_{\Delta}\left(\bar{a}_{\beta}, M, \mathfrak{A}\right)$

(c) $\|M\| \leq \mu$.

1A) Let $\mathfrak{A}$ be $\left(\aleph_{0}, \Delta\right.$, per)-unstable mean that $(\mathfrak{A}, \Delta)$ is $\aleph_{0}$-unstable; here per stands for perfect.

2) In part (1) and (1A) we add "weakly" if we weaken clause (b) to

$(b)^{-} \operatorname{tp}_{\Delta}\left(\bar{a}_{\eta}, M, \mathfrak{A}\right) \neq \operatorname{tp}_{\Delta}\left(\bar{a}_{\nu}, M, \mathfrak{A}\right)$ for $\eta \neq \nu$ from $X$

(so if $\Delta$ is closed under negation there is no difference); in part (1), $X=\lambda$ and in part (1A), $X={ }^{\omega} 2$.

3) We use $\left(\mu_{0}, \Delta, x, \mathbb{Q}\right)$ where $\mathbb{Q}$ is a forcing notion iff the example is found in $\mathbf{V}^{\mathbb{Q}}$ such that usually $M$ is in $\mathbf{V}$ and we add an additional possibility if $x=$ per then $M \in \mathbf{V}$ and $X=\left({ }^{\omega} 2\right)^{\mathbf{V}}$ (here per stands for perfect).

4) We may replace "a forcing notion $\mathbb{Q}$ " by a family $\mathfrak{K}$ of forcing notions (e.g. the family of c.c.c. ones) meaning: for at least one of them.

$5)$ We replace unstable by stable for the negation.

Observation 2.8. If $\Delta$ is closed under negation, then $\mathfrak{A}$ is weakly $\left(\aleph_{0}, \Delta, \lambda\right)$ unstable iff $\mathfrak{A}$ is $\left(\aleph_{0}, \Delta, \lambda\right)$-unstable. 


\section{§3. RANK AND INDISCERNIBILITY}

Definition 3.1. Let $(\mathfrak{A}, \Delta)$ be a $\kappa$-candidate. Let $1 \leq m \in \mathbb{N}$ and assume $D$ is a $\kappa^{+}$-complete filter on ${ }^{m} \mathfrak{A}$; (or a filter on some $\mathbf{I} \subseteq{ }^{m} \mathfrak{A}$, then we interpret it as $\left\{\mathbf{J} \subseteq{ }^{m} \mathfrak{A}: \mathbf{J} \cap \mathbf{I} \in D\right\}$; writing $\operatorname{rk}_{\lambda}^{m}$ we mean $\operatorname{rk}_{D}^{m}$ means $D=\left\{\mathbf{I} \subseteq{ }^{m} \mathfrak{A}:{ }^{m} \mathfrak{A} \backslash \mathbf{I}\right.$ has cardinality $\leq \lambda\}$; similarly $\mathrm{rk}_{<\lambda}^{n}$ ).

For $\mathbf{I} \subseteq{ }^{m} \mathfrak{A}, \mathbf{I} \neq \emptyset \bmod D$ we define $\operatorname{rk}_{D}^{m}(\mathbf{I})=\operatorname{rk}_{D}^{m}(\mathbf{I}, \Delta, \mathfrak{A})$, an ordinal or infinity or -1 by defining for any ordinal $\alpha$ when $\operatorname{rk}_{D}^{m}(\mathbf{I}) \geq \alpha$ by induction on $\alpha$.

Case 1: $\alpha=0$.

$\operatorname{rk}_{D}^{m}(\mathbf{I}) \geq \alpha$ iff $\mathbf{I} \neq \emptyset \bmod D$.

Case 2: $\alpha$ limit.

$\operatorname{rk}_{D}^{m}(\mathbf{I}) \geq \alpha$ iff $\operatorname{rk}_{D}^{m}(\mathbf{I}) \geq \beta$ for every $\beta<\alpha$.

Case 3: $\alpha=\beta+1$.

$\operatorname{rk}_{D}^{m}(\mathbf{I}) \geq \alpha$ iff $(\mathrm{a})+(\mathrm{b})$ holds where

(a) if $\mathbf{I}=\cup\left\{\mathbf{I}_{i}: i<\kappa\right\}$ then for some $i<\kappa$ we have $\operatorname{rk}_{D}^{m}\left(\mathbf{I}_{i}\right) \geq \beta$

(b) we can find $\bar{\varphi}(\bar{x}, \bar{b}) \in \Phi_{\mathfrak{A}, \Delta}^{m}$ and $\mathbf{I}_{0}, \mathbf{I}_{1} \subseteq \mathbf{I}$ such that $\operatorname{rk}_{D}^{m}\left(\mathbf{I}_{\ell}\right) \geq \beta$ and $\bar{a} \in \mathbf{I}_{\ell} \Rightarrow \mathfrak{A}=\varphi_{\ell}(\bar{a}, \bar{b})$ for $\ell=0,1$.

Observation 3.2. Assume $(\mathfrak{A}, \Delta)$ is a $\kappa$-candidate.

1) If $\alpha \leq \beta$ are ordinals and $\operatorname{rk}_{D}^{m}(\mathbf{I}) \geq \beta \underline{\text { then }} \operatorname{rk}_{D}^{m}(\mathbf{I}) \geq \alpha$.

2) $\operatorname{rk}_{D}^{m}(\mathbf{I}) \in \operatorname{Ord} \cup\{-1, \infty\}$ is well defined (for $\mathbf{I} \subseteq{ }^{m} \mathfrak{A}$ ).

3) If $\mathbf{I}_{1} \subseteq \mathbf{I}_{2} \subseteq \mathfrak{A}$ then $\operatorname{rk}_{D}^{m}\left(\mathbf{I}_{1}\right) \leq \operatorname{rk}_{D}^{m}\left(\mathbf{I}_{2}\right)$.

Proof. Trivial.

Theorem 3.3. The following are equivalent if $2^{\aleph_{0}} \geq \kappa^{+}$and $(\mathfrak{A}, \Delta)$ is a $\kappa$ candidate:

(a) $\operatorname{rk}_{\kappa}^{m}\left({ }^{m} \mathfrak{A}\right) \geq \kappa^{+}$for some $m$

(b) $\mathfrak{A}$ is $\left(\aleph_{0}, \Delta\right)$-unstable, see Definition 1.4(3)

(c) $\mathfrak{A}$ is $\left(\aleph_{0}, \Delta, \kappa^{+}\right)$-unstable, see Definition 2.7(1)

(d) $\mathrm{rk}_{\kappa}^{m}\left({ }^{m} \mathfrak{A}\right)=\infty$ for some $m$

(e) $\mathfrak{A}$ is not $\left(\aleph_{0}, \Delta\right)$-stable, see Definition 1.4(2)

(f) the assumption $\boxplus$ of 2.4.

Proof. We first prove $(a) \Rightarrow(b) \Rightarrow(c) \Rightarrow(d) \Rightarrow(a)$, then $(c) \Rightarrow(e), \neg(d) \Rightarrow \neg(e)$ and lastly $(a) \Rightarrow(f) \Rightarrow(b)$; clearly this is sufficient.

$(a) \Rightarrow(b)$.

Let $\mathscr{P}_{\alpha}=\left\{\mathbf{I} \subseteq{ }^{m} \mathfrak{A}: \mathrm{rk}_{\kappa}^{m}(\mathbf{I}) \geq \alpha\right\}$ for $\alpha<\kappa^{+}$and apply Claim 2.4.

$\underline{(b) \Rightarrow(c)}$ : Trivial recalling Definition 1.4, 2.7 as we are assuming $2^{\aleph_{0}} \geq \kappa^{+}$.

$(c) \Rightarrow(d)$ :

Let $A \subseteq \mathfrak{A}$ be countable and $\left\{\bar{a}_{\alpha}: \alpha<\kappa^{+}\right\} \subseteq{ }^{m} \mathfrak{A}$ exemplify that $\mathfrak{A}$ is $\left(\aleph_{0}, \Delta, \kappa^{+}\right)$-unstable.

Now without loss of generality

$(*)$ if $\bar{b} \subseteq A, \varphi(\bar{x}, \bar{y}) \in \Delta$ then 
- $\left\{\alpha<\kappa^{+}: \mathfrak{A} \models \varphi\left(\bar{a}_{\alpha}, \bar{b}\right)\right\}$ is either unbounded or is empty

- $\left\{\alpha<\kappa^{+}: \mathfrak{A} \models \neg \varphi\left[\bar{a}_{\alpha}, \bar{b}\right]\right\}$ is either unbounded or is empty.

Now let $\mathscr{P}=\left\{\left\{\bar{a}_{\alpha}: \alpha \in S\right\}: S \subseteq \kappa^{+}\right.$is unbounded $\}$. Now we can prove by induction on $\alpha$ that $\mathbf{I} \in \mathscr{P} \Rightarrow \operatorname{rk}_{D}^{m}(\mathbf{I}) \geq \alpha$ recalling $2.7(1)(\mathrm{b})$.

$\underline{(d) \Rightarrow(a)}$ : Trivial.

$\neg(e) \Rightarrow \neg(c)$ :

Let $m, M$ and $\left\langle\bar{a}_{\alpha}: \alpha<\kappa^{+}\right\rangle$be as in Definition 2.7(1). Let $\chi$ be large enough and let $N \prec(\mathscr{H}(\chi), \in)$ be of cardinality $\kappa$ such that $\kappa+1 \subseteq N$ hence $M \subseteq N$ and $\mathfrak{A}, \Delta, M$ and $\left\langle\bar{a}_{\alpha}: \alpha<\kappa^{+}\right\rangle$belong to $N$. So by 1.6(3) for every $\alpha<\kappa^{+}$for some c $\in N$ we have (**) of $\boxplus$ of Definition 1.4(2). As $\|N\|=\kappa$ necessarily for some $\mathbf{c}_{*} \in N$ we have $S=\left\{\alpha<\kappa^{+}: \mathbf{c}_{\alpha}=\mathbf{c}_{*}\right\}$ has cardinality $\kappa^{+}$. Choose $\alpha \neq \beta$ from $S$ and get a contradiction to the choice of the $\left\langle\bar{a}_{\alpha}: \alpha<\kappa^{+}\right\rangle$.

$\neg(d) \Rightarrow \neg(e):$

We have to prove $\neg(e)$, i.e. $\mathfrak{A}$ is $\left(\Delta, \aleph_{0}\right)$-stable, see Definition 1.4(2). We use $3.3(\mathrm{c})$.

So we can find $m<\omega, N \prec(\mathscr{H}(\chi), \in), \mathfrak{A} \in N, \kappa+1 \subseteq N$ and $\|N\|=\kappa$ and $\bar{a} \in{ }^{m} \mathfrak{A}$ and it suffices to find a function $\mathbf{c}$ in 1.4(2). Now let $\mathscr{Z}:=\left\{\mathrm{rk}_{\kappa}^{m}(\mathbf{I}): \mathbf{I} \in \mathscr{B}\right\}$ where $\mathscr{B}=\left\{\mathbf{I}: \mathbf{I} \in N\right.$ is a non-empty subset of ${ }^{m} \mathfrak{A}$ to which $\bar{a}$ belongs $\}$. Now this family $\mathscr{B}$ is not empty (as ${ }^{m} \mathfrak{A} \in \mathscr{B}$ ) hence $\mathscr{Z} \neq \emptyset$.

Of course, $\infty \notin \mathscr{Z}$ by our present assumption, i.e. $\neg(d)$. Hence $\beta=\min (\mathscr{Z})$ is a well defined ordinal, so by the definition of $\mathscr{Z}$ there is $\mathbf{I} \in \mathscr{B}$ such that $\operatorname{rk}_{\kappa}^{m}(\mathbf{I})=\beta$; clearly $\mathbf{I} \in N$ by the choice of $\mathscr{B}$, hence also $\beta \in N$. Now why $\operatorname{rk}_{\kappa}^{m}(\mathbf{I}) \ngtr \alpha:=\beta+1$ ? By Definition 3.1, case 3, clause (a) or clause (b) there fail.

Case 1: Clause (a) fails.

Then there is a sequence $\overline{\mathbf{I}}=\left\langle\mathbf{I}_{i}: i<\kappa\right\rangle$ of subsets of $\mathbf{I}$ with union $\mathbf{I}$ such that $i<\kappa \Rightarrow \operatorname{rk}_{\kappa}^{m}\left(\mathbf{I}_{i}\right)<\beta$, clearly $\overline{\mathbf{I}} \in \mathscr{H}(\chi)$ hence as $\mathbf{I}, \beta \in N$ without loss of generality $\overline{\mathbf{I}} \in N$. As $\kappa \subseteq N$ necessarily $i<\kappa \Rightarrow \mathbf{I}_{i} \in N$ and obviously $i<\kappa \Rightarrow \mathbf{I}_{i} \subseteq \mathbf{I} \subseteq{ }^{m} \mathfrak{A}$.

Lastly, as $\bar{a} \in \mathbf{I}$ necessarily there is $j<\kappa$ such that $\bar{a} \in \mathbf{I}_{j}$. So $\mathbf{I}_{j}$ witness $\operatorname{rk}_{\kappa}^{m}\left(\mathbf{I}_{j}\right) \in \mathscr{Z}$ and as said above $\operatorname{rk}_{\kappa}^{m}\left(\mathbf{I}_{j}\right)<\beta$, so contradicting the choice of $\beta$ as $\min (\mathscr{Z})$.

$\underline{\text { Case 2: }}$ Clause (b) (of case 3 of Definition 3.1) fails (for our $\alpha, \beta, \mathbf{I}$ ).

So

$\odot_{1}$ for every $\bar{\varphi}(\bar{x}, \bar{b}) \in \Phi_{\mathfrak{A}, \Delta}^{m}$ we can choose $\iota<2$ such that $\operatorname{rk}_{\kappa}^{m}\left(\left\{\bar{a}^{\prime} \in \mathbf{I}: \mathfrak{A} \models\right.\right.$ $\left.\left.\varphi_{\iota}\left[\bar{a}^{\prime}, \bar{b}\right]\right\}\right)<\beta$.

So there is a function $\mathbf{t} \in N$ with domain $\Phi_{\mathfrak{A}, \Delta}^{m}$ and range $\subseteq\{0,1\}$ such that

$\odot_{2}$ if $\bar{\varphi}(\bar{x}, \bar{b}) \in \Phi_{\mathfrak{A}, \Delta}^{m}$ then $\mathbf{t}=\mathbf{t}(\bar{\varphi}(\bar{x}, \bar{b}))$ satisfies $\operatorname{rk}_{\kappa}^{m}\left(\mathbf{I}_{\bar{\varphi}(\bar{x}, \bar{b}), \mathbf{t}}\right)<\beta$ where

$\odot_{3} \quad \mathbf{I}_{\bar{\varphi}(\bar{x}, \bar{b}), \mathbf{t}}=\left\{\bar{a}^{\prime} \in \mathbf{I}: \mathfrak{A} \models \varphi_{\mathbf{t}}\left[\bar{a}^{\prime}, \bar{b}\right]\right\}$.

However

$\odot_{4}$ if $\bar{\varphi}(\bar{x}, \bar{b}) \in \Phi_{\mathfrak{A}, \Delta}^{m} \cap N$ and $\mathbf{t}<2$ and $\mathfrak{A} \models \varphi_{\mathbf{t}}[\bar{a}, \bar{b}]$ then

(a) $\mathbf{I}_{\bar{\varphi}(\bar{x}, \bar{b}), \mathbf{t}} \in \mathscr{B}$

(b) $\operatorname{rk}_{\kappa}^{m}\left(\mathbf{I}_{\bar{\varphi}(\bar{x}, \bar{b}), \mathbf{t}}\right) \in \mathscr{Z}$ 
(c) $\operatorname{rk}_{\kappa}^{m}\left(\mathbf{I}_{\bar{\varphi}(\bar{x}, \bar{b}), \mathbf{t}}\right)=\beta$

(d) $\mathbf{t} \neq \mathbf{t}(\bar{\varphi}(\bar{x}, \bar{b}))$.

[Why? For clause (a): as $\bar{\varphi}(\bar{x}, \bar{b}) \in N$ and $\mathbf{I} \in \mathscr{B} \subseteq N$ clearly $\mathbf{I}_{\bar{\varphi}(\bar{x}, \bar{b}), \mathbf{t}} \in N$ and it is $\subseteq \mathbf{I} \subseteq{ }^{m} \mathfrak{A}$. Also, $\bar{a} \in \mathbf{I}_{\bar{\varphi}(\bar{x}, \bar{b}), \mathbf{t}}$ by the assumption of $\odot_{4}$, together $\mathbf{I}_{\bar{\varphi}(\bar{x}, \bar{b}), \mathbf{t}} \in \mathscr{B}$ as required. Hence by the definition of $\mathscr{Z}$ also clause (b) holds. But by 3.2(3) we have $\operatorname{rk}_{\kappa}^{m}\left(\mathbf{I}_{\bar{\varphi}(\bar{x}, \bar{b}), \mathbf{t}}\right) \leq \operatorname{rk}_{\kappa}^{m}(\mathbf{I})=\beta$ so by the choice of $\beta$ as $\min (\mathscr{Z})$ we get equality, i.e. clause (c) holds. Recalling $\odot_{2}$ we get clause (d).]

Now by $\odot_{4}$ (as $\mathbf{t}$ is a function from $N$ ), we are done proving $\neg(e)$.

$\underline{(a) \Rightarrow(f):}$

For $\alpha<\kappa^{+}$let $\mathscr{P}_{\alpha}=\left\{\mathbf{I} \subseteq{ }^{m} \mathfrak{A}: \operatorname{rk}_{K}^{m}(\mathbf{I}) \geq \alpha\right\}$ and recalling Definition 3.1 it is easy.

$\underline{(f) \Rightarrow(b)}:$ By 2.4 .

Conclusion 3.4. 1) The property $" \mathrm{rk}_{\kappa}^{m}(\mathfrak{A})=\infty$ " is preserved by forcing.

2) If $\operatorname{rk}_{\kappa}^{m}\left({ }^{m} \mathfrak{A}\right)=\infty$ then for some $\mathscr{U}_{1} \subseteq \kappa$ coding the definitions of $\mathfrak{A}$ and of the $\kappa$-Suslin definitions of every $\varphi(\bar{x}) \in \Delta$, and $\mathscr{U}_{2} \subseteq$ Ord every forcing extension of $\mathbf{L}\left[\mathscr{U}_{1}, \mathscr{U}_{2}\right]$ satisfies $\operatorname{rk}_{\kappa}^{m}\left({ }^{m} \mathfrak{A}\right)=\infty$.

Proof. 1) By Theorem 3.3 it suffices to prove clause (b) there $\left((\mathfrak{A}, \Delta)\right.$ is $\left(\aleph_{0}, \Delta\right)$ unstable) is preserved, but by Theorem 3.3, i.e. $(a) \Rightarrow(f)$ there, the assumption of 2.4 holds but its conclusion says that " $(\mathfrak{A}, \Delta)$ is $\left(\aleph_{0}, \Delta\right)$-unstable" holds also in forcing extensions of $\mathbf{V}$.

2) Similarly.

Definition 3.5. If $p$ is a $\left(\Delta_{1}, m\right)$-type over $A$ in $\mathfrak{A}$ (i.e. a set of formulas $\varphi(\bar{x}, \bar{a})$ with $\varphi(\bar{x}, \bar{y}) \in \Delta_{1}, \bar{a} \subseteq A$ ), we let (may write $\mu$ instead of $<\mu^{+}$)

$$
\begin{aligned}
\operatorname{rk}_{<\lambda}^{m}\left(p, \Delta_{1}, \mathfrak{A}\right)=\operatorname{Min}\left\{\operatorname{rk}_{<\lambda}^{m}\left(\bigwedge_{\ell<n} \varphi_{\ell}\left({ }^{m} \mathfrak{A}, \bar{b}_{\ell}\right), \Delta_{1}, \mathfrak{A}\right):\right. & n<\omega \\
& \text { and } \left.\varphi_{\ell}\left(\bar{x}, \bar{b}_{\ell}\right) \in p \text { for } \ell<n\right\} .
\end{aligned}
$$

Observation 3.6. 1) If $p \subseteq q$ (or just $q \vdash p)$ are $(\Delta, m)$-types in $\mathfrak{A}$ then $\operatorname{rk}_{<\lambda}^{m}(q, \Delta, \mathfrak{A}) \leq$ $\mathrm{rk}_{<\lambda}^{m}(p, \Delta, A)$.

2) If $q$ is a $(\Delta, m)$-type in $\mathfrak{A} \underline{\text { then }}$ for some finite $p \subseteq q$ we have

$$
\operatorname{rk}_{<\lambda}^{m}(q, \Delta, \mathfrak{A})=\operatorname{rk}_{<\lambda}^{m}(p, \Delta, \mathfrak{A})
$$

hence

$$
p \subseteq r \subseteq q \Rightarrow \operatorname{rk}_{<\lambda}^{m}(r, \Delta, \mathfrak{A})=\operatorname{rk}_{<\lambda}^{m}(p, \Delta, \mathfrak{A}) .
$$

Proof. Obvious by the definitions.

Claim 3.7. Assume $\kappa^{+} \leq 2^{\aleph_{0}}$. In 3.3 we can add

(g) for some $\mu<\operatorname{cf}(\lambda)$ and, of course, $\kappa<\operatorname{cf}(\lambda), \lambda \leq 2^{\aleph_{0}}$ the pair $(\mathfrak{A}, \Delta)$ is $(\mu, \Delta, \lambda)$-unstable

(h) like (g) for every such $\mu, \lambda$

(i) $(\mathfrak{A}, \Delta)$ is not $\aleph_{0}$-stable. 
Proof. $\neg(i) \Rightarrow \neg(c)$.

Let $\left.\overline{M \prec(\mathscr{H}(\chi)}, \in,<_{\chi}^{*}\right)$ be countable such that $(\mathfrak{A}, \Delta) \in M$ and $m<\omega$. For every $\bar{a} \in{ }^{m} \mathfrak{A}$ there is a function $\mathbf{c}_{\bar{a}} \in M$ from $\Phi_{(\mathfrak{A}, \Delta)}^{m}$ to $\{0,1\}$ as in Definition 1.4. So if $\bar{a}_{i} \in{ }^{m} \mathfrak{A}$ for $i<\kappa^{+}$then for some $i<j<\kappa^{+}$we have $\mathbf{c}_{\bar{a}_{i}}=\mathbf{c}_{\bar{a}_{j}}$ because $M$ is countable hence for no $\bar{\varphi}(\bar{x}, \bar{b}) \in \Phi_{(M, \Delta)}^{m}$ do we have $\varphi_{0}(\bar{x}, \bar{b}) \in \operatorname{tp}_{\Delta}\left(\bar{a}_{i}, M, \mathfrak{A}\right), \varphi_{1}(\bar{x}, \bar{b}) \in \operatorname{tp}_{\Delta}\left(\bar{a}_{j}, M, \mathfrak{A}\right)$. So clearly $(c)$ fails.

$(i) \Rightarrow(c)$.

Fix $\left(\mathscr{H}\left(\chi_{0}\right), \in,<_{\chi}^{*}\right)$ and let

$$
\begin{array}{ll}
\mathscr{S}_{0}=\left\{M \prec\left(\mathscr{H}\left(\chi_{0}\right), \in,<_{\chi}^{*}\right):\right. & \mathfrak{A} \in M \text { and }\|M\|=\kappa \\
& \text { and } \kappa+1 \subseteq M\} .
\end{array}
$$

For $m<\omega$ and $\mathbf{I} \subseteq{ }^{m} \mathfrak{A}$ let $\mathscr{J}_{\mathbf{I}}=\mathscr{J}[\mathbf{I}]$ be the family of $\mathscr{S} \subseteq \mathscr{S}_{0}$ such that: we can find $\left\langle F_{x}, \mathbf{c}_{x}: x \in \mathscr{H}(\chi)\right\rangle$ (a witness) such that:

$(\alpha) \mathbf{c}_{x}: \Phi_{\mathfrak{A}, \Delta}^{m} \rightarrow\{0,1\}$

(ß) $F_{x}:{ }^{\omega>}(\mathscr{H}(\chi)) \rightarrow \mathscr{H}(\chi)$

$(\gamma)$ if $M \in \mathscr{S}_{0}$ is closed under $F_{x}$ for $x \in M$ then for every $\bar{a} \in \mathbf{I}$ for some $y \in M, \mathbf{c}_{y}$ is a witness for $\operatorname{tp}\left(\bar{a}_{M}, M \cap \mathfrak{A}, \mathfrak{A}\right)$, see Definition $1.4(2)$.

Clearly $\mathscr{J}_{\mathbf{I}}$ is a normal ideal on $\mathscr{S}_{0}$. Also if " $m<\omega \Rightarrow \mathscr{S}_{0} \in \mathscr{J}\left[{ }^{m} \mathfrak{A}\right]$ " then increasing $\chi$ we get the desired result. Toward contradiction assume that $m<\omega$ and $\mathscr{S}_{0} \notin \mathscr{J}\left[{ }^{m} \mathfrak{A}\right]$ and let $\mathscr{P}$ (i.e. $\mathscr{P}_{\alpha}=\mathscr{P}$ for $\alpha<\kappa^{+}$) be the family of $\mathbf{I} \subseteq{ }^{m} \mathfrak{A}$ such that $\mathscr{S}_{0} \notin \mathscr{J}_{\mathbf{I}}$.

We now finish by 2.4 once we prove

$\circledast$ if $\mathbf{I} \in \mathscr{P}$ then for some $\bar{\varphi}(\bar{x}, \bar{b}) \in \Phi_{\mathfrak{A}, \Delta}^{m}$ for each $\ell<2$ the set $\mathbf{I}_{\bar{\varphi}(x, \bar{b})}^{\ell}$ is $\left\{\bar{a} \in \mathbf{I}: \mathfrak{A} \models \varphi_{\ell}(\bar{a}, \bar{b})\right\}$ belong to $\mathscr{P}$.

If not, for every $\bar{\varphi}(\bar{x}, \bar{b}) \in \Phi_{\mathfrak{A}, \Delta}^{m}$ there is $\ell=\mathbf{c}[\bar{\varphi}(\bar{x}, \bar{b})]<2$ and $\left\langle\left(F_{x}^{\bar{\varphi}(\bar{x}, \bar{b})}, \mathbf{c}_{x}^{\bar{\varphi}(\bar{x}, \bar{b})}\right)\right.$ : $x \in \mathscr{H}(\chi)\rangle$ witnessing $\mathscr{S}_{0} \in \mathscr{J}\left[\mathbf{I}_{\bar{\varphi}}^{\ell}\right]$.

Define $\left(F_{y}, \mathbf{c}_{y}\right)$ for $y \in \mathscr{H}(\chi)$ by: if $y=\langle x, \bar{\varphi}(\bar{x}, \bar{b})\rangle$ then $F_{y}=F_{x}^{\bar{\varphi}(\bar{x}, \bar{b})}, c_{y}=$ $\mathbf{c}_{x}^{\bar{\varphi}(\bar{x}, b)}$, otherwise $\mathbf{c}$.

Clearly we can find $M \in \mathscr{S}_{0}$ such that

$\circledast_{1}$ if $\bar{\varphi}(\bar{x}, \bar{b}) \in \Phi_{\mathfrak{A}, \Delta}^{m} \cap M$ and $x \in M$ then $M$ is closed under $F_{x}^{\bar{\varphi}(\bar{x}, \bar{b})}$

$\circledast_{2}$ for some $\bar{a} \in{ }^{m} \mathfrak{A}$, no $\mathbf{c}_{y}, y \in M$ defines $\operatorname{tp}_{\Delta}(\bar{a}, M \cap \mathfrak{A}, \mathfrak{A})$, in the sense of $1.4(2)$.

But $\mathbf{c}$ does it! So we are done. Alternatively, note $(i)=(c)$ so we can quote 3.3.

$\underline{(h) \Rightarrow(g)}$.

Obvious.

$(g) \Rightarrow(d)$.

Like $(c) \Rightarrow(d)$.

$(c) \Rightarrow(h)$.

Repeat the proof of " $(c) \Rightarrow(d)$ " in 3.3. 
Theorem 3.8. Assume that $(\mathfrak{A}, \Delta)$ is a $\kappa$-candidate and is $\aleph_{0}$-stable or just $\mu$ stable.

For some $\xi<\kappa^{+}$we have: if $\lambda \geq \mu, m<\omega, A \subseteq \mathfrak{A},|A| \leq \lambda$ and $\bar{a}_{\alpha} \in{ }^{m} \mathfrak{A}$ for $\alpha<\lambda^{+\xi}$ then for some $S \subseteq \lambda^{+\xi}$ of cardinality $\lambda$ the sequence $\left\langle\bar{a}_{\alpha}: \alpha \in S\right\rangle$ is a $\Delta$-indiscernible sequence over $A$ in $\mathfrak{A}$.

Remark 3.9. On getting indiscernible sets, see 0.12, 2.1 .

Proof. First, fix $A, \lambda, \mu$ and assume the conclusion fails.

For $\xi<\kappa^{+}$let

$$
\begin{aligned}
\mathscr{P}_{\xi}=\{\mathbf{I}: \quad & \mathbf{I} \subseteq m^{m} \mathfrak{A} \text { has cardinality } \geq \lambda^{+\xi} \text { and no } \mathbf{I}^{\prime} \subseteq \mathbf{I} \text { of cardinality } \\
& \left.\lambda \text { is } \mathbf{I}^{\prime} \text { a } \Delta \text {-indiscernible sequence over } A \text { in } \mathfrak{A}\right\} .
\end{aligned}
$$

So by Claim 2.4 it suffices to check the demands $\boxplus(a)-(e)$ there are satisfied by $\overline{\mathscr{P}}=\left\langle\mathscr{P}_{\xi}: \xi<\kappa^{+}\right\rangle$defined above and $\Phi:=\Phi_{(\mathfrak{A}, \Delta)}^{m}$.

Now clause (a) holds by by our assumptions (and the choice of $\Phi$ ).

Clause (b) holds by the choice of $\mathscr{P}_{\xi}$ and clause (c) holds by our assumption toward contradiction. Also clause (e) there is trivial because $\mathbf{I} \in \mathscr{P}_{\xi} \wedge \mathbf{I}^{\prime} \subseteq \mathbf{I} \cap\left|\mathbf{I}^{\prime}\right| \geq$ $\kappa^{+\zeta} \Rightarrow \mathbf{I}^{\prime} \in \mathscr{P}_{\zeta}$.

So we are left with proving clause (d) there, for this (as $\mathscr{P}_{\xi}$ increases with $\xi$ ) it suffices to prove the following:

$\circledast$ if $\lambda^{+\xi}$ is regular, $\xi>\zeta, \bar{a}_{\alpha} \in{ }^{m} \mathfrak{A}$ for $\alpha<\lambda^{+\xi}$ and $S \subseteq \lambda^{+\xi}$ is stationary then (a) or (b) where:

(a) for some club $E$ of $\lambda,\left\langle\bar{a}_{\alpha}: \alpha \in S \cap E\right\rangle$ is a $\Delta$-indiscernible set over $A$ in $\mathfrak{A}$

(b) for some $\bar{\varphi}(\bar{x}, \bar{b}) \in \Phi^{m}(\mathfrak{A}, \Delta)$ the sets $\mathbf{I}_{0}, \mathbf{I}_{1}$ have cardinality $\geq \lambda^{+\zeta}$ where $\mathbf{I}_{\ell}=\left\{\bar{a} \in \mathbf{I}: \mathfrak{A} \models \varphi_{\ell}[\bar{a}, \bar{b}]\right\}$ for $\ell=0,1$.

So we fix $\xi, \mathbf{I}$ and $\zeta$ as in $\circledast$ and we shall prove that $(\mathrm{a})$ of $\circledast$ or $(\mathrm{b})$ of $\circledast$ holds.

Now

$\oplus$ for some $m<\omega$ and club $E_{n}^{*}$ of $\lambda^{+\xi}$ we have:

(i) $\left\langle\bar{a}_{\alpha}: \alpha \in S \cap E_{n}^{*}\right\rangle$ is $(\Delta, m)$-end extension indiscernible sequence over $A$ in $\mathfrak{A}$

(ii) for no club $E^{\prime} \subseteq E_{n}^{*}$ of $\lambda^{+\xi}$ is $\left\langle\bar{a}_{\alpha}: \alpha \in S \cap E^{\prime}\right\rangle$ a sequence which is $(\Delta, m+1)$-end extension indiscernible.

[Why? If for every $m$ there is a club $E_{m}^{*}$ of $\lambda^{+\xi}$ such that clause (i) of $\oplus$ holds, then $E=\cap\left\{E_{n}^{*}: n\right\}$ is a club of $\lambda^{+\xi}$ satisfying clause (i) of $\circledast$ for every $m$.

Hence $E$ is a club of $\lambda^{+\xi}$ such that $\left\langle\bar{a}_{\alpha}: \alpha \in S \cap E\right\rangle$ is $\Delta$-indiscernible sequence over $A$ in $\mathfrak{A}$, so as required in clause (a) of $\circledast$, (hence is as required in the theorem), but we are assuming there is no such set, so for some $m$ there is no such $E_{m}^{*}$. But for $m=0$ there is such $E_{m}^{*}$ : the club $E_{0}^{*}:=\lambda^{+\xi}$. Together for some $m$ there is a club $E_{m}^{*}$ such that clause $(i)$ of $\oplus$ holds for this $m$ but there is no such club for $m+1$. So $\oplus$ holds indeed.]

By claim 1.7(3) there is a club $E$ of $\lambda$ and $\left\langle f_{k}: k<\omega\right\rangle$ as there, (in fact, only $f_{m}, f_{m+1}$ are used), let $S_{\gamma}^{*}=\left\{\alpha \in S: \alpha>\gamma\right.$ and $\left.f_{m+1}(\alpha)=\gamma\right\}$, and let $W_{m+1}$ be $\left\{\gamma: S_{\gamma}^{*}\right.$ is stationary $\}$.

Clearly there is a $E_{*}$ such that: 
(a) $E_{*}$ is a club of $\lambda^{+\xi}$

(b) $E_{*} \subseteq E \cap E_{m}^{*}$

(c) if $\gamma<\lambda^{+\xi}$ but $\gamma \notin W_{n+1}$ then $S_{\gamma}^{*}$ is disjoint to $E_{*} \backslash(\gamma+1)$ and even to $E_{*}$

(d) if $\sup \left(W_{n+1}\right)<\lambda^{+\xi}$ then $\sup \left(W_{n+1}\right)<\min \left(E_{*}\right)$

Case 1: $W_{n+1}=\emptyset$

As $f_{n+1}$ is a pressing down function on $E$, a club of $\lambda^{+\xi}$ this is impossible.

Case 2: $W_{n+1}$ is a singleton, say $\left\{\gamma_{*}\right\}$

In this case $f_{m+1}$ is constant on $S \cap E_{*}$ hence clearly $\left\langle\bar{a}_{\alpha}: \alpha \in S \cap E_{*}\right\rangle$ contradiction to the choice of $m$.

Case 3: $W_{n}$ has at least two elements

Let $\gamma_{1}<\gamma_{2}$ belongs to $W_{n+1}$, applying 1.10 we get the desired result.

Well, we still have a debt: why $\xi$ does not depend on $(A, \mu, \lambda)$ ? So assume for each $\xi,\left(A_{\xi}, \mu_{\xi}, \lambda_{\xi}, \xi, \mathscr{I}_{\xi}\right)$ forms a counterexample. Now formalizing the above and we quote " $\psi$ has a model such that $\left(Q^{M},<^{M}\right)$ is not well ordered when $\psi \in \mathbb{L}_{\kappa^{+}, \aleph_{0}}$ has a model $M_{\xi},\left(Q^{M_{\xi}},<^{M_{\xi}}\right)=(\xi, \alpha)$ for arbitrarily large $\xi<\kappa^{+"}$, see Keisler [Kei71].

Claim 3.10. Assume $(\mathfrak{A}, \Delta)$ is a $\kappa$-candidate and it is $\left(\aleph_{0}, \Delta\right)$-unstable, see Claim 3.3 and Definition 1.4(3). Then $\mathfrak{A}$ is not categorical 1 , even "under $2^{\aleph_{0}}=\aleph_{1}$ ", see $0.5(3 A)$.

Remark 3.11. Of course, we can get stronger versions: many models.

Proof. Let $\bar{x}=\bar{x}_{m}, \bar{\varphi}_{\nu}=\left(\varphi_{\nu, 0}\left(\bar{x}, y_{\nu}\right), \varphi_{\nu, 1}(\bar{x}, \bar{y})\right) \in \Phi_{(\mathfrak{A}, \Delta)}^{m}$ for $\nu \in \omega>2$ and $\left\langle\bar{b}_{\nu}\right.$ : $\left.\nu \in{ }^{\omega>} 2\right\rangle,\left\langle\bar{a}_{\eta}: \eta \in{ }^{\omega} 2\right\rangle$ be as in Definition 1.4(3).

Without loss of generality this holds absolutely, i.e. if $\mathbb{P}$ is a forcing extension, and $\eta \in\left({ }^{\omega} 2\right)^{\mathbf{V}[\mathbb{P}]}$ then we can choose $\bar{a}_{\eta}$.

Let $\mathbb{Q}$ be the forcing of adding $\aleph_{1}$ Cohens, $\bar{\eta}=\left\langle\eta_{\alpha}: \alpha<\aleph_{1}\right\rangle$ the generic and $\mathbb{P}$ be trivial and easily $\mathfrak{A}=\mathfrak{A}^{\mathbf{V}}, \mathfrak{A}^{\mathbf{V}[\mathbb{Q}]}$ are not isomorphic: let $\underset{\tilde{\mathbf{V}}}{F}$ be a $\mathbb{Q}$-name and assume toward contradiction $p \Vdash " \underset{\sim}{F}$ is an isomorphism from $\mathfrak{A}^{\tilde{\mathbf{V}}[\mathbb{Q}]}$ onto $\mathfrak{A}^{\mathbf{V}}$ " where $p \in \mathbf{G}$ and $\mathbf{G} \subseteq \mathbb{Q}$ is generic over $\mathbf{V}$. So for some $\alpha(*)<\aleph_{1},\left\langle\underset{\sim}{F}\left\lceil\left({\underset{\sim}{\nu}}_{\nu}\right): \nu \in \omega>2\right\rangle\right.$ depend just on $\left\langle\eta_{\alpha}: \alpha<\alpha(*)\right\rangle$ so in $\mathbf{V}[\bar{\eta} \mid \alpha(*)]$ we can compute it, so $\underset{\sim}{F}\left(\eta_{\alpha(*)}\right)$ can have no possible value, contradiction. 


\section{$\S 4$. Concluding Remarks}

We try to expand on justifying 0.7 , that is the connection to categoricity of $\psi \in \mathbb{L}_{\aleph_{1}, \aleph_{0}}$ and how much "categoricity in $\lambda$ " depends on $\lambda$.

First we may consider more relatives of 0.9 .

Definition 4.1. For a definition $\mathfrak{A}$ of a $\tau$-model (usually with a set of elements a definable set of reals) and $\lambda=\lambda^{\aleph_{0}}$; we say $\mathfrak{A}$ is $\mathfrak{K}$-categorical ${ }_{3}$ in $\lambda \underline{\text { when }}$ : if $\mathbb{P}_{2}=\mathbb{P}_{0} \times \mathbb{P}_{1}$ are forcing notions from $\mathfrak{K}$ such that $\left|\mathbb{P}_{\ell}\right|=\lambda$ and $\vdash_{\mathbb{P}_{\ell}} " 2^{\aleph_{0}}=\lambda "$, $\underline{\text { then }}$ there is a forcing notion $\mathbb{P}_{3} \in \mathfrak{K}$ such that $\mathbb{P}_{2} \lessdot \mathbb{P}_{3}$, moreover $\mathbb{P}_{2} \leq_{\mathfrak{K}} \mathbb{P}_{3}$ and $\frac{{ }_{\mathbb{P}_{3}}}{} " \mathfrak{A} \mathbf{V}\left[\mathbb{P}_{0}\right], \mathfrak{A}^{\mathbf{V}\left[\mathbb{P}_{1}\right]}$ are isomorphic".

It may be helpful to first analyze (Cohen, $\lambda$ )-categoricity $y_{2}$, assuming $0^{\#}$ exists, so $\mathfrak{K}=\{\mathbb{Q}$ : for some cardinal $\mu, \mathbb{Q}$ is the forcing notion of adding $\mu$ Cohen reals $\}$ order by $\mathbb{P} \leq_{\mathfrak{K}} \mathbb{Q}$ iff $\mathbb{P} \lessdot \mathbb{Q}$ and $\mathbb{P}, \mathbb{P} / \mathbb{Q}$ are from $\mathfrak{K}$. Hence $\mathbb{P}_{\ell}$ is adding $\lambda$ Cohen reals for $\ell=1,2$ and $\mathbb{P}_{3}$ is (isomorphic to) adding $(\lambda+\lambda)$-Cohen reals. So let $\bar{\eta}_{1}{ }^{\wedge} \bar{\eta}_{2}$ be generic for adding $(\lambda+\lambda)$-Cohens to $\mathbf{V}$ and $\bar{\eta}_{\ell}=\left\langle\eta_{\alpha}^{\ell}: \alpha<\lambda\right\rangle$ for $\ell=1,2$. (So $\bar{\eta}_{\ell}$ is generic over $\mathbf{L}$ and for notational simplicity we may assume that the $\mathbf{V}=\mathbf{L}$ so $\tau$ and the definition of $\mathfrak{A}$ belongs to $\mathbf{L})$.

For $\ell=1,2$ and $u \subseteq \lambda$ let $\mathfrak{A}_{\ell, u}$ be $\mathfrak{A}^{\mathbf{V}\left[\bar{\eta}_{\ell}\lceil u]\right.}$ and the forcing notions $\mathbb{P}_{\ell, u} \lessdot \mathbb{P}_{\ell}$ for $\ell=1,2,3$ be naturally defined. From the categoricity assumption it follows that without loss of generality $\mathbb{P}_{3}$ is adding $(\lambda+\lambda+\lambda)$-Cohen reals $\mathbb{P}_{\ell}=\mathbb{P}_{3,[\lambda \ell, \lambda \ell+\lambda]}$, and there is a $\mathbb{P}_{3}$-name $\underset{\sim}{f}$ such that

$(*) \Vdash_{\mathbb{P}_{3}}$ "ff is an isomorphism from $\mathfrak{A}\left[\bar{\eta}_{1}\right]$ onto $\mathfrak{A}\left[\bar{\eta}_{2}\right]$ ".

So in $\mathbf{V}$, for some function $F:{ }^{\omega} \lambda \rightarrow \lambda$ we have

$(*)$ if $u \subseteq \lambda$ is infinite and closed under $F$ then $f_{n}=f \mid \mathfrak{A}_{1, u}$ is a $\mathbb{P}_{1, u}$-name and is an isomorphism from $\mathfrak{A}_{1, u}$ onto $\mathfrak{A}_{2, u}$.

For $u \subseteq \lambda$ let $c \ell_{F}(u)$ be the closure of $u$ by $F$. Now if $\lambda \geq\left(2^{\aleph_{0}}\right)^{+n}$ and $n \geq m$

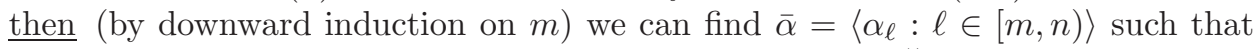
$\overline{k \in}[m, n] \Rightarrow \alpha_{k} \notin c \ell_{F}\left(\left\{\alpha_{\ell}: \ell \in[m, n)\right.\right.$ but $\left.\left.\ell \neq k\right\} \cup\left(2^{\aleph_{0}}\right)^{+m}\right)$. So letting for $v \subseteq n, u_{v}=c \ell_{F}\left(\left\{\alpha_{k}: k \in v\right\} \cup\left(2^{\aleph_{0}}\right)\right)$ we have:

(*) (a) $f_{u_{v}}$ is an isomorphism from $\mathfrak{A}_{1, u_{v}}$ onto $\mathfrak{A}_{2, u_{v}}$ increasing with $v$ for $v \subseteq n$

(b) if $f(1) \subseteq v(2) \subseteq n$ then $f_{u_{v(1)}} \subseteq f_{u_{v(2)}}$

(c) if $v \subseteq n, k<n$ and $k \notin v$ then $\alpha_{k} \notin u_{v}$.

This seems to suggest the stability of $\left(\mu, \mathscr{P}^{-}(n)\right)$-diagrams as in [She83b]. We expect that for every finite sequence $\bar{a}$ from $\mathfrak{A}_{1, n}$ its type over $\cup\left\{\mathfrak{A}_{1, u}: u \in \mathscr{P}^{-}(n)\right\}$ is definable in a suitable sense.

Of course, we may well have to consider also (assuming for transparency $2^{\aleph_{0}}=$ $\left.\aleph_{1}\right)$ in addition to the $\mathfrak{A}_{1, u_{v}}$ of cardinality $\aleph_{1}$ also countable approximation $\mathfrak{A}_{1, u_{v}}^{\prime}$.

Also more seriously, maybe the stability of $\left(\mathscr{P}^{-}(n), 2^{\aleph_{0}}\right)$-diagrams will not be enough, because for $\lambda>\left(2^{\aleph_{0}}\right)^{+\omega}$ the $F$-closure of $u,|u|=\left(2^{\aleph_{0}}\right)^{+\omega}$ has bigger cardinality. In this case maybe this stops after $\omega_{1}$ steps. 


\section{REFERENCES}

[HS90] Bradd T. Hart and Saharon Shelah, Categoricity over $P$ for first order $T$ or categoricity for $\phi \in \mathcal{L}_{\omega_{1} \omega}$ can stop at $\aleph_{k}$ while holding for $\aleph_{0}, \cdots, \aleph_{k-1}$, Israel J. Math. 70 (1990), no. 2, 219-235, arXiv: math/9201240. MR 1070267

[Jec03] Thomas Jech, Set theory, Springer Monographs in Mathematics, Springer-Verlag, Berlin, 2003, The third millennium edition, revised and expanded.

[Kei71] H. Jerome Keisler, Model theory for infinitary logic. logic with countable conjunctions and finite quantifiers, Studies in Logic and the Foundations of Mathematics, vol. 62, North-Holland Publishing Co., Amsterdam-London, 1971.

${ }^{\left[S^{+}\right] \quad S . ~ S h e l a h ~ e t ~ a l ., ~ T b a, ~ I n ~ p r e p a r a t i o n . ~ P r e l i m i n a r y ~ n u m b e r: ~ S h: F 1134 . ~}$

[She] Saharon Shelah, Introduction and Annotated Contents, arXiv: 0903.3428 introduction of [Sh:h].

[She83a] Classification theory for nonelementary classes. I. The number of uncountable models of $\psi \in L_{\omega_{1}, \omega}$. Part A, Israel J. Math. 46 (1983), no. 3, 212-240. MR 733351

[She83b] Classification theory for nonelementary classes. I. The number of uncountable models of $\psi \in L_{\omega_{1}, \omega}$. Part B, Israel J. Math. 46 (1983), no. 4, 241-273. MR 730343

[She84] Un co-א-Souslin relations, Israel J. Math. 47 (1984), no. 2-3, 139-153. MR 738165

[She90] _ Classification theory and the number of nonisomorphic models, 2nd ed., Studies in Logic and the Foundations of Mathematics, vol. 92, North-Holland Publishing Co., Amsterdam, 1990. MR 1083551

[She94] Cardinal arithmetic, Oxford Logic Guides, vol. 29, The Clarendon Press, Oxford University Press, New York, 1994. MR 1318912

[She99a] —, Borel sets with large squares, Fund. Math. 159 (1999), no. 1, 1-50, arXiv: math/9802134. MR 1669643

[She99b] _ Borel Whitehead groups, Math. Japon. 50 (1999), no. 1, 121-130, arXiv: math/9809198. MR 1710476

[She09a] _ Categoricity in abstract elementary classes: going up inductively, 2009, arXiv: math/0011215 Ch. II of [Sh:h].

[She09b] _ Classification theory for abstract elementary classes, Studies in Logic (London), vol. 18, College Publications, London, 2009. MR 2643267

[She09c] _ Universal Classes: Axiomatic Framework [Sh:h], 2009, Ch. V (B) of [Sh:i].

[She11] , Polish algebras, shy from freedom, Israel J. Math. 181 (2011), 477-507, arXiv: math/0212250. MR 2773054

[She14] _ Strongly dependent theories, Israel J. Math. 204 (2014), no. 1, 1-83, arXiv: math/0504197. MR 3273451

[SV21] Saharon Shelah and Andrés Villaveces, The Hart-Shelah example, in stronger logics, Ann. Pure Appl. Logic 172 (2021), no. 6, 102958, 23, arXiv: math/0404258. MR 4216281

Einstein Institute of Mathematics, Edmond J. Safra Campus, Givat Ram, The HeBrew University of Jerusalem, Jerusalem, 91904, Israel, and, Department of Mathematics, Hill Center - Busch Campus, Rutgers, The State University of New Jersey, 110 Frelinghuysen Road, Piscataway, NJ 08854-8019 USA

Email address: shelah@math.huji.ac.il

$U R L:$ http://shelah.logic.at 